\title{
On Strictly Enforced Mass Conservation Constraints for Modeling the Rainfall-Runoff Process
}

\author{
Jonathan M. Frame ${ }^{1,2}$, Frederik Kratzert ${ }^{3}$, Hoshin V. Gupta ${ }^{4}$, Paul Ullrich ${ }^{5}$, and Grey S. Nearing 5,6 \\ ${ }^{1}$ University of Alabama, Tuscaloosa, AL, United States \\ ${ }^{2}$ National Water Center, National Oceanic and Atmospheric Administration, Tuscaloosa, AL, United States \\ ${ }^{3}$ Google Research, Vienna, Austria \\ ${ }^{4}$ The University of Arizona, Tucson, AZ, United States \\ ${ }^{5}$ University of California Davis, Department of Land, Air \& Water Resources, Davis, CA, United States \\ ${ }^{6}$ Google Research, Mountain View, CA, United States
}

Correspondence: jmframe@ crimson.ua.edu

\begin{abstract}
It has been proposed that conservation laws might not be beneficial for accurate hydrological modeling due to errors in input (precipitation) and target (streamflow) data, and this might explain why deep learning models (which are not based on enforcing closure) can out-perform catchment-scale conceptual and process-based models at predicting streamflow. We test this hypothesis using physics-informed machine learning and find that: (1) enforcing closure in the rainfall-runoff mass balance does appear to harm the overall skill of hydrological models, (2) deep learning models learn to account for spatiotemporally variable biases in data, however (3) this "closure" effect accounts for only a small fraction of the difference in predictive skill between deep learning and conceptual models.
\end{abstract}

Keywords - Deep learning, rainfall-runoff, physics-informed machine learning, LSTM, mass conservation, large sample hydrology, CAMELS, water balance 


\section{Introduction}

Deep learning (DL) models are becoming the standard benchmark for predictive hydrologic modeling in the current literature because of their high accuracy relative to conceptual and process-based (PB) models (Nearing et al., 2020c), as well as their ability to extrapolate to new locations (Kratzert et al., 2019a) and extreme events (Frame et al., 2021a). There has been a recent push to combine deep learning with physical theory to (i) gain better process understanding, and (ii) improve predictive accuracy, especially under out-of-sample conditions (Jia et al., 2020; Reichstein et al., 2019; Shen et al., 2021; Willard et al., 2021). There have been several recent attempts to build hybrid PB-DL models (sometimes referred to "physics-informed" or "theory-guided", e.g., Bennett and Nijssen, 2021; Karniadakis et al., 2021; Pelissier et al., 2019; Daw et al., 2020; Tsai et al., 2020; Zhao et al., 2019; Jiang et al., 2020; Xie et al., 2021; Hoedt et al., 2021; Nearing et al., 2020a). We therefore think it is important to take a step back and explore if (and what) basic components of physical theory might actually be beneficial for hydrologic prediction.

In this paper we test one hypothesis in particular: we use physics-informed machine learning to explore the longstanding assumption that mass conservation should be the foundation of hydrological models. The first physical law introduced formally by Chow et al. (1988, equation 1.3.5) (a standard introductory hydrology textbook) is:

$d S / d t=I(t)-Q(t)$,

where the change of a system's mass storage (S) with respect to time (t) is equal to total mass input (I) minus total mass output (Q). This is the first physical constraint placed on the transfer function between inputs and output of a hydrological system (i.e., Chow et al., 1988, equation 1.3.1).

While conservation laws are considered to be a fundamental truth about (classical scale) systems in our physical world, it is not necessarily the case that this makes them a proper or useful foundation for either understanding or modeling watershed systems. This distinction is motivated by Beven (2020), who proposed that the closure problem might explain the poor performance of conceptual and physically-based (PB) hydrology models relative to DL:

"given the epistemic uncertainties in water and energy balances, then this [conservation constraints] might not necessarily be advantageous in obtaining better DL predictions if, for example, the observational data do not themselves provide consistent mass and energy balance closure".

In other words, conceptual and PB models typically demand a degree of closure that may not necessarily be achievable given sparse and error-prone observation data, and (Beven hypothesized that) the superior performance of DL might be due to its ability to learn and account for consistent error structures present in the input-output data. In practice, PB models sometimes account for error prone data with pre- and post-processing, as well as data assimilation, however pre- and post-processing is not necessary when using DL, as these steps can be learned directly from training data (e.g., Frame et al., 2021b).

The proposal explains poor rainfall-runoff model calibration and performance as being a consequence of so-called "disinformation" in data (e.g., Beven et al., 2008; Beven and Westerberg, 2011; Sivapalan et al., 2003). In addition to the observational uncertainty present in data used for driving and evaluating models, there is also uncertainty regarding what actually constitute the true physical inputs and losses from a hydrologic system - for example, mass contributions to the system through natural springs and anthropogenic water resources can come from outside of the watershed "boundary" and are not often directly observable or represented in the available data set. Beven's hypothesis is that these types of effects might explain the relative accuracy of DL streamflow models, due to their not being constrained to conserve mass.

In this paper, we place a bound on this "closure" effect (i.e., on the information loss due to enforcing closure over error-prone data), and show two things: 
1. DL is able to learn and account for systematic (but spatiotemporally dynamic) errors in data, and

2. the closure effect does not explain the majority of the performance gap between PB models and DL models of streamflow.

Here we test the ability and performance of the mass conserving MC-LSTM to performing its namesake task (mass conservation), by assessing the long-term bias of predicted runoff in a large-sample dataset (Gupta and Nearing, 2014). To be clear, we are not questioning whether hydrologic processes in the real world are governed by the physical concept of mass conservation. What we are questioning is whether a testable, scale-relevant theory of watersheds should be based on this principle. Alternatively, it is possible that no successful scalerelevant theory of watersheds has been developed to-date because the fundamental conceptual basis for a "watershed" is itself incorrect; the typical "fixed catchment control-volume" represented by our watershed delineations with closed internal states cannot represent mesoscale storm-system scales and groundwater aquifer scales.

\section{Methods}

60 We designed an experiment to test the hypothesis proposed by Beven (2000) that the lack of mass conservation in DL rainfall-runoff models explains the difference in skill relative to PB models that are constrained by closure. The basic experiment is as follows. We use two meteorological data sets, one with a large, nonlinear, and location-specific bias and one without such a bias, to benchmark three models: (i) a standard PB model calibrated per-basin, (ii) a standard DL model trained regionally (over all basins, not per-basin), and (iii) a physicsinformed DL model that is constrained to enforce mass conservation trained regionally (in the same way as the standard DL model). Our goal is to understand how much of the difference in skill between the PB and DL models can be accounted for by forcing closure on biased data.

\subsection{Data}

Several recent modeling studies used open community data sets and consistent training/test procedures that allow for results to be directly comparable (Kratzert et al., 2019b, 2021; Klotz et al., 2021; Gauch et al., 2021b, a; Newman et al., 2017; Frame et al., 2021b, a). We continue that practice here. Specifically, we used the Catchment Attributes and Meteorological Large Sample (CAMELS) data set curated by the US National Center for Atmospheric Research (NCAR) (Newman et al., 2015; Addor et al., 2017). The CAMELS data set consists of daily meteorological and discharge data from 671 catchments in CONUS ranging in size from $4 \mathrm{~km}^{2}$ to $25,000 \mathrm{~km}^{2}$ that have largely natural flows and long streamflow gauge records (1980-2008). Newman et al. (2017) developed CAMELS as a data set for community model benchmarking and by excluding basins with (i) large discrepancies between different methods of calculating catchment area, and (ii) areas larger than 2,000 $\mathrm{km}^{2}$. This results in the large-sample (Gupta and Nearing, 2014) data set with 531 basins that has been used by all of the benchmarking studies cited above. In the current study, we had to omit one of these 531 basins due to a data constraint that will be explained below in Section 2.3.

CAMELS includes daily discharge data from the USGS Water Information System, which are used as training and evaluation targets. CAMELS also includes three daily meteorological forcing data products (Daymet, NLDAS, Maurer) that are used as model inputs. CAMELS also includes several static catchment attributes related to soils, climate, vegetation, topography, and geology (Addor et al., 2017) that are used as input features to the DL models. We used the same input features (meteorological forcings and static catchment attributes) that are listed in Table 1 by Kratzert et al. (2019b).

We used Daymet and NLDAS for this project. The reason that we used these two meteorological forcing data sets is because Daymet exhibits a large bias in the Eastern US relative to USGS streamflow data, while NLDAS does not. This bias can be seen clearly in some 

different regions of CONUS. The regions were delineated according the United States Geological Survey (USGS) Water Resources Regions outlined in Water-Supply Paper 2294 (USGS, 1987). This includes 18 distinct regions, but only 17 of which have enough CAMELS basins for meaningful statistics (leaving out Souris-Red-Rainy, hydrologic unit code 09).

\subsection{Models}

\subsubsection{Conceptual and process models}

The conceptual model that we used as a benchmark was the Sacramento Soil Moisture Accounting model (SAC-SMA) with SNOW-17 and a unit hydrograph routing function. This is the model used by (Newman et al., 2017) as a basis for standardized benchmarking with the CAMELS data set, however we re-calibrated SAC-SMA to be consistent with our training/test splits. We used the Python-based SACSMA code and calibration package developed by (Nearing et al., 2020b), which uses the SpotPy calibration library (Houska et al., 2019). SAC-SMA was calibrated separately at each of the 531 CAMELS basins using the three train/test splits outlined in Section 2.2.3.

We also benchmarked the U.S. National Water Model (NWM), which is a process(physics)-based model using the NOAA National Water Model CONUS Retrospective Dataset (https://registry.opendata.aws/nwm-archive/ accessed December 2021). We present the results of the NWM benchmarks in Appendix C, rather than the main body of this paper because (i) the NWM is only available for NLDAS forcing, and (ii) we are not able to calibrate the NWM to match our other models, so the NWM results aren't directly comparable. A complete description of the NWM is provided in Appendix C along with a complete set of figures.

\subsubsection{Deep learning models}

The Long Short Term Memory (LSTM) network is the current state-of-the-art model for predicting streamflow at the watershed scale. The LSTM is a recurrent neural network with an explicit state space, and explicit controls on input-state and state-output relationships, as well as explicit controls on memory timescales, which makes it suitable for at least many dynamical systems applications. The LSTM does not enforce conservation laws, which means that there is potential for predicted runoff to violate Equation 1.

The Mass-Conserving LSTM (MC-LSTM) is also a recurrent neural network with an explicit state space and explicit input-state and stateoutput relationships. The internal calculations of the MC-LSTM ensure mass-conservation between any number of inputs (here precipitation) and outputs (here streamflow). In reality, precipitation and streamflow are not the only fluxes of water into or out of a catchment. The MCLSTM accounts for unobserved sinks (e.g., evapotranspiration, aquifer recharge and anthropogenic water resources) using a subset of cell states to accumulate mass that does not translate to streamflow.

Appendixes A and B provide details of the LSTM and MC-LSTM, respectively.

\subsubsection{Training}

We used daily meteorological forcing data and static catchment attributes data as inputs features for the LSTM and MC-LSTM, and we used daily streamflow records as training targets with a normalized squared-error loss function that does not depend on basin-specific mean discharge (i.e., large and/or wet basins are not over-weighted in the loss function):

$\mathrm{NSE}^{*}=\frac{1}{B} \sum_{b=1}^{B} \sum_{n=1}^{N} \frac{\left(\widehat{y}_{n}-y_{n}\right)^{2}}{(s(b)+\epsilon)^{2}}$, 
where $B$ is the number of basins, $N$ is the number of samples (days) per basin $B, \widehat{y}_{n}$ is the prediction for sample $n(1 \leq n \leq N), y_{n}$ is the corresponding observation, and $s(b)$ is the standard deviation of the discharge in basin $b(1 \leq b \leq B)$, calculated from the training period (see Kratzert et al., 2019b).

We trained both the standard LSTM and the MC-LSTM using the same training and test procedures outlined by Kratzert et al. (2019b). Both models were trained for 30 epochs using sequence-to-one prediction to allow for randomized, small minibatches. We used a minibatch size of 256 and, due to sequence-to-one training, each minibatch contained (randomly selected) samples from multiple basins. The standard LSTM had 128 cell states and a 365-day sequence length. Input and target features for the standard LSTM were pre-normalized by removing bias and scaling by variance. For the MC-LSTM the inputs were split between auxiliary, which were pre-normalized, and the mass input (in our case precipitation), which was not pre-normalized. Gradients were clipped to a global norm (per minibatch) of 1 . Heteroscedastic noise was added to training targets (resampled at each minibatch) with standard deviation of 0.005 times the value of each target datum. We used an Adam optimizer with a fixed learning rate schedule; the initial learning rate of $1 \mathrm{e}-3$ was decreased to $5 \mathrm{e}-4$ after 10 epochs and $1 \mathrm{e}-4$ after 25 epochs. Biases of the LSTM forget gate were initialized to 3 so that gradient signals persisted through the sequence from early epochs. The MC-LSTM used the same hyperparameters as the LSTM except that it used only 64 cell states, which was found to perform better for this model (see, Hoedt et al., 2021). Note that the memory states in an MC-LSTM are fundamentally different than those of the LSTM due to the fact that they are physical states with physical units instead of purely information states.

Both the LSTM and MC-LSTM were trained on data from 531 CAMELS catchments simultaneously. The train/test period split was the same split used in previous studies (Kratzert et al., 2019b, 2021; Hoedt et al., 2021). In this case, the training period included nine water years from October 1, 1999 through September 30, 2008, and the test period included ten water years 1990-1999 (i.e., from October 1, 1989 through September 30,1999). This train/test split was used only to ensure that the models trained here achieved similar performance compared with previous studies. Appendix C includes an analysis of a different time period (the train period included water years 1981-1995, and the test period included water years1996-2014), which was chosen to overlap with the NWM-Rv2 retrospective run.

\subsection{Performance metrics}

We report two sets of performance metrics. The first set are standard benchmarking metrics that we report for two reasons: (i) to show that the models perform similarly with previous benchmarking studies, and (ii) to allow us to demonstrate a distinction between model performance and consistency of long-term mass balance. The second set of metrics are related to long-term streamflow biases, and allow us to test our primary hypothesis. These metrics are described in the following two subsections.

\subsubsection{Standard performance metrics}

We benchmarked all models using the same set of performance metrics that were used in previous CAMELS studies (Kratzert et al., 2019b, a, 2021; Gauch et al., 2021a; Klotz et al., 2021). A full list of these metrics is given in Table 1. Each of the metrics was calculated for each basin separately on the whole test period for the training/test splits described in Section 2.2.3 (the test period consists of water years 1990-1999).

\subsubsection{Long term mass balance}

We also conducted a long-term mass balance analysis using the absolute mass bias error for each basin:

total absolute mass bias $=\frac{\mid \sum \text { obs. } Q-\sum \text { sim. } Q \mid}{\sum \text { obs. } Q}$ 
Table 1. Overview of performance benchmarking evaluation metrics for hydrological models. The notation of the original publications is kept.

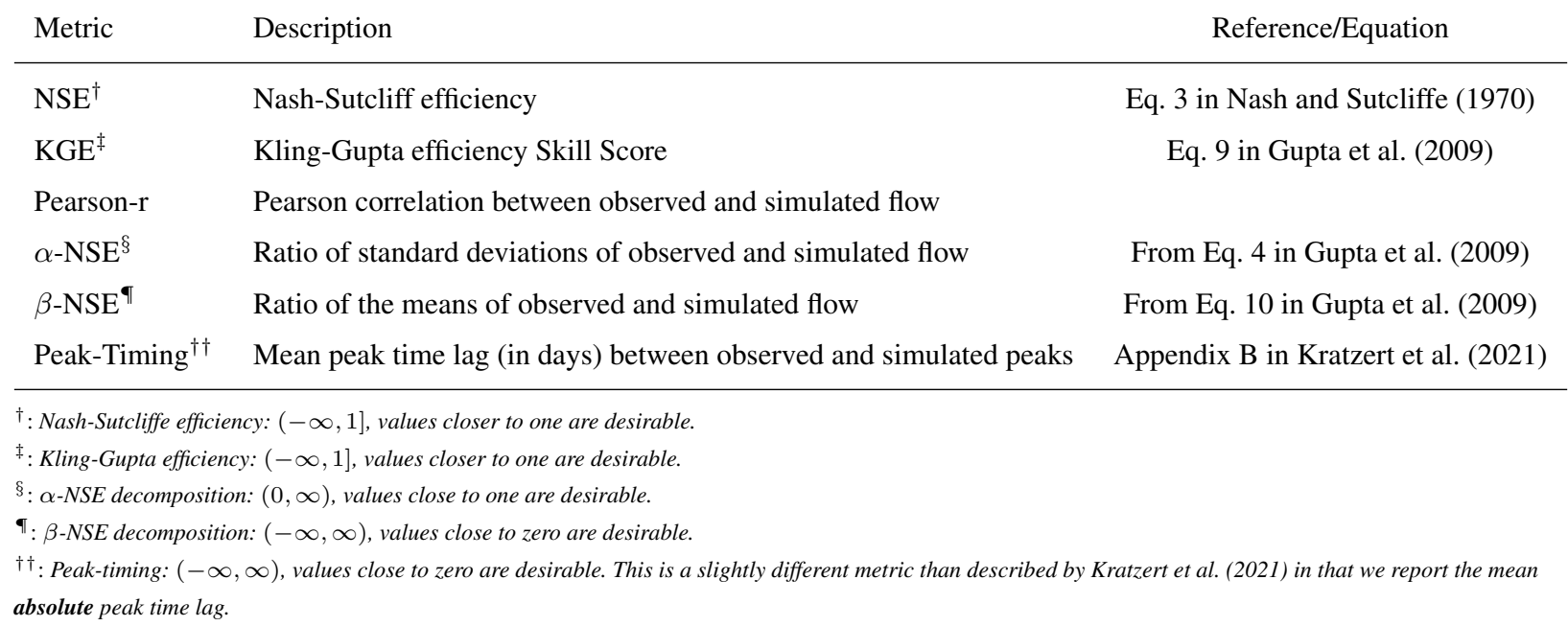

where $\mathrm{Q}$ is the mass flux of streamflow. The positive and negative mass bias error for each basin is calculated as

positive mass bias $= \begin{cases}x=\frac{\sum \text { obs. } Q-\sum \text { sim. } Q}{\sum o b s . Q}, & \text { if } x>0 \\ 0, & \text { otherwise }\end{cases}$

and

negative mass bias $=\left\{\begin{array}{ll}x=-\frac{\sum o b s \cdot Q-\sum \text { sim. } Q}{\sum o b s . Q}, & \text { if } x<0 \\ 0, & \text { otherwise }\end{array}\right.$.

155 We used these metrics to provide a general measure the ability of each model to close the mass balance between precipitation and streamflow). These metrics require a continuous observation record, however only 530 of the 531 CAMELS benchmarking basins satisfy this for the test period of water years 1990-1999.

\subsubsection{Measuring information loss from modeling constraints}

Following the discussion by (Nearing and Gupta, 2015), we anticipate an ordering of information content like:

$H_{\text {streamflow }} \geq I_{\text {inputdata }} \geq I_{L S T M} \geq I_{M C-L S T M} \geq I_{S A C-S M A}$

$\mathrm{H}$ indicates the total entropy of whatever target data we are trying to predict (here a hydrograph in an individual basin). There is some amount of information in the input data (meteorological forcings and basin attributes), however the data processing inequality Cover and Thomas (2005, equation 2.122, page 35) indicates that information is lost by any model which means that any model prediction contains less than, or equal to, information about the target data than is contained in the raw inputs (see Nearing and Gupta, 2015, for further discussion). Finally, we hypothesize that the constraints in the MC-LSTM (mass conservation) and the conceptual SAC-SMA model will mean that these two models provide less information than the LSTM. It is important to point out that the latter two terms of Equation/Inequality 6 are only 
hypotheses - it is possible that adding constraints to a trained model (either a neural network or a calibrated conceptual model) will improve performance. We consider this unlikely, since adding constraints to a DL model serves only to restrict the space of functions that the model can emulate, however it is always possible that regularization will help avoid local minima during training, or otherwise compensate for limited information content of training data.

We quantified this (hypothesized) chain of inequalities using two difference metrics. The first metric is the standard mutual information (MI) metric calculated by histograms with 100 bins:

$$
M I(U, V)=\sum_{i=1}^{|U|} \sum_{j=1}^{|V|} \frac{\left|U_{i} \bigcap V_{j}\right|}{N} \log \frac{N\left|U_{i} \bigcap V_{j}\right|}{\left|U_{i}\right|\left|V_{j}\right|}
$$

where $U$ is the observed streamflow, $V$ is the simulated streamflow and $N$ is the number of records. Mutual information obeys the data processing inequality, so that the first and second terms of Equation/Inequality 6 apply strictly. We calculated the MI in two ways: (1) at each basin individually for a distribution of values, and (2) using all of the flows from all basins combined for an overall MI score that does not account for distinctions between basins.

We also report the skill score outlined by Knoben et al. (2019) based on KGE metrics:

$$
K G E_{\text {skillscore }}=\frac{K G E_{\text {model }}-K G E_{\text {baseline }}}{1-K G E_{\text {baseline }}}
$$

where the skill score compares the performance of a candidate model with a baseline. This lets us draw an intuitive connection between the benchmarking metrics in Section 2.3.1 and Equation 6, however we do omit proof that the KGE obeys the data processing inequality, and therefore the relationship is only intuitive. The first level of constraint that we test is the strictly enforced mass conservation in the MC-LSTM and SAC-SMA. This is analogous to the third inequality in Equation 6, and in this case $K G E_{m o d e l}$ and $K G E_{\text {baseline }}$ in Equation 8 are the MC-LSTM and the LSTM, respectively. The second level of constraint is the conceptualization of the watershed as implemented by SAC-SMA model architecture (remember that SAC-SMA is calibrated to long data records in each basin, which removes most parameter uncertainty). This is analogous to the third term in Equation/Inequality 6, and in this case $K G E_{\text {model }}$ and $K G E_{\text {baseline }}$ in Equation 8 are SAC-SMA and the LSTM, respectively (we use the LSTM instead of the MC-LSTM as the baseline in order to plot a direct comparison between information lost by the MC-LSTM and SAC-SMA).

\subsection{Conditionality of the modelling analysis}

Uncertainty in this experiment comes from three primary sources: data, models, and training. These sources are analogous to standard sources of uncertainty in most hydrology modeling studies: data, model structure, and model parameters (training is analogous to calibration).

The hypothesis that we are testing is related to understanding relationships between data and model uncertainty. Our objective is to understand how different models deal with uncertainty in data. We do not explicitly represent uncertainty in data (e.g., probabilistically), because our experiment does not require this for testing the hypothesis. We treat training/calibration uncertainty by using an ensemble of models. This is approach is discussed explicitly for DL models by (Kratzert et al., 2019a), and for the SAC-SMA model by (Newman et al., 2015). Our analysis in Section 3.2 includes a box-and-whisker plot showing the mean, standard deviation and outliers of each performance metric. We also include a complete (second) set of results of the same analysis on a different time period in Appendix C, with results that are nearly identical, indicating that our results are not the result of an anomalous time period.

Our models and training are consistent with previous studies. Benchmarking results like what are reported in Section 3.1 have been 
Table 2. Median performance metrics (plus or minus the $95 \%$ confidence interval) across 530 basins calculated on the test period $1990-1999$ with two separate forcing products.

\begin{tabular}{l|rrr|rrr}
\hline \multirow{2}{*}{ Metric } & \multicolumn{3}{|c}{ Daymet forcing } & \multicolumn{3}{c}{ NLDAS forcing } \\
\hline NSE & LSTM & MC-LSTM & SAC-SMA & LSTM & MC-LSTM & SAC-SMA \\
KGE & $0.77 \pm-0.02$ & $0.76 \pm-0.01$ & $0.65 \pm-0.03$ & $0.74 \pm-0.01$ & $0.74 \pm-0.01$ & $0.67 \pm-0.02$ \\
Pearson-r & $0.76 \pm-0.02$ & $0.76 \pm-0.02$ & $0.59 \pm \mathrm{n} / \mathrm{a}$ & $0.74 \pm-0.02$ & $0.74 \pm-0.02$ & $0.68 \pm-0.02$ \\
Alpha-NSE & $0.89 \pm-0.01$ & $0.88 \pm-0.01$ & $0.83 \pm \mathrm{n} / \mathrm{a}$ & $0.88 \pm-0.01$ & $0.87 \pm-0.01$ & $0.83 \pm-0.01$ \\
Beta-NSE & $-0.04 \pm-0.01$ & $-0.84 \pm-0.01$ & $0.76 \pm-0.02$ & $0.81 \pm-0.02$ & $0.81 \pm-0.02$ & $0.78 \pm-0.02$ \\
Peak-Timing & $0.3 \pm-0.03$ & $0.3 \pm-0.03$ & $0.38 \pm-0.06$ & $0.32 \pm-0.03$ & $0.31 \pm-0.03$ & $0.41 \pm-0.06$ \\
\hline
\end{tabular}

large-sample studies for all models, which provides a degree of confidence about the modeling results in general. We included $5 / 95 \%$ confidence intervals of the summary statistics, and these are relatively low given our large sample size.

\section{Results}

\subsection{Model performance}

Table 2 provides performance metrics (Section 2.3.1 for the LSTM, MC-LSTM and SAC-SMA model simulations over the test period (water years 1990-1999). Most of these scores are broadly equivalent to the metrics for the same models reported by other studies Kratzert et al. (2019b, e.g.,). More importantly, these metrics allow us to test the hypothesis that explicit mass conservation degrades performance (as a reminder, this hypothesis was proposed by (Beven, 2020)). What we are looking for in these metrics is that either all the mass conserving models perform worse than the non-constrained LSTM, which would support the hypothesis that mass conservation is detrimental to models, or that the MC-LSTM with an explicit mass conserving constraint does as well or better than the LSTM, which would indicate that the problem with the conceptual model is not a matter of enforcing closure over erroneous data.

Results show similar average performance between the LSTM and MC-LSTM, however there were largely small differences. The LSTM had a higher KGE score in 323 basins, with an average difference of 0.06, and the MC-LSTM had a higher KGE score in 208 basins, also with an average difference of 0.06. In general, the median performance metrics of the two models were broadly comparable. Both models were, on average, better across all metrics than SAC-SMA. This suggests that enforcing closure does not explain the differences between data-based and process-based models.

\subsection{Long-term cumulative discharge}

Figure 1 shows the cumulative density functions (CDFs) of long-term cumulative discharge from the 530 CAMELS basins from the models during the 1989-1999 test period. The LSTM, MC-LSTM and SAC-SMA all have a similar total mass bias with the NLDAS forcing. SAC-SMA has the lowest negative mass error, but the highest positive mass error. The LSTM has the highest negative mass error, but the lowest positive mass error. The MC-LSTM is generally in between the LSTM and SAC-SMA. Overall, the LSTM and MC-LSTM predicted streamflows that result in more accurate long-term cumulative discharge than the calibrated SAC-SMA model. The LSTM and the MC-LSTM 
performed roughly similarly on NLDAS, and MC-LSTM slightly outperformed the LSTM on Daymet. With Daymet forcing SAC-SMA's streamflow predictions are biased towards a very high positive mass error.
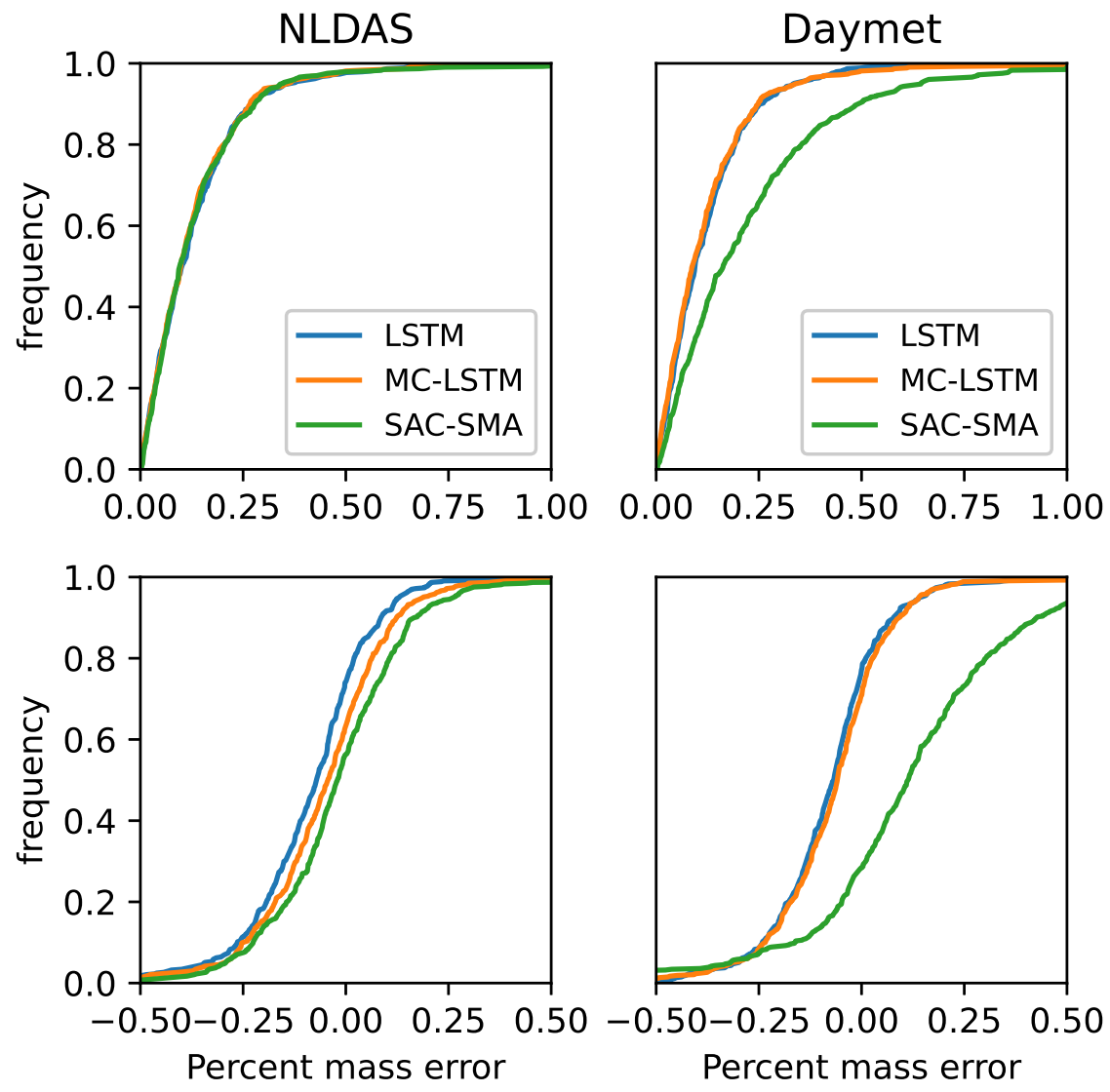

Figure 1. Distribution of mass balance error across the 530 basins. Top: Cumulative distribution curves of the absolute mass error from models forced with NLDAS (left) and Daymet (right). Bottom: Cumulative distributions of mass error from models forced with NLDAS (left) and Daymet (right).

Figure 2 shows the long term positive or negative mass biases distributed across the Contiguous United States (CONUS) from for the three models with both Daymet and NLDAS forcings. The result of the SAC-SMA simulation with Daymet forcings shows a clear positive mass bias error in the eastern half of CONUS. The result of the SAC-SMA simulation with NLDAS forcings shows a mix of positive and negative mass bias throughout CONUS. The LSTM and the MC-LSTM look relatively similar, to each other and for both NLDAS and Daymet forcing. This Central CONUS (CenCon) region (i.e., Missouri, Arkansas-White-Red and Texas-Gulf) is generally tough to predict, with conceptual, physical and deep learning models. SAC-SMA also shows a negative mass bias pattern in the same central CONUS region, though to a lesser spatial extent and higher magnitude, with Daymet forcings, but not so much with NLDAS forcings.

Figure 3 shows the mass bias errors for the model runs with Daymet forcings in box and whisker plots for the U.S. Water Resources Regions. SAC-SMA shows a very high mass balance error in the eleven eastern regions, but does much better in the western regions. The 

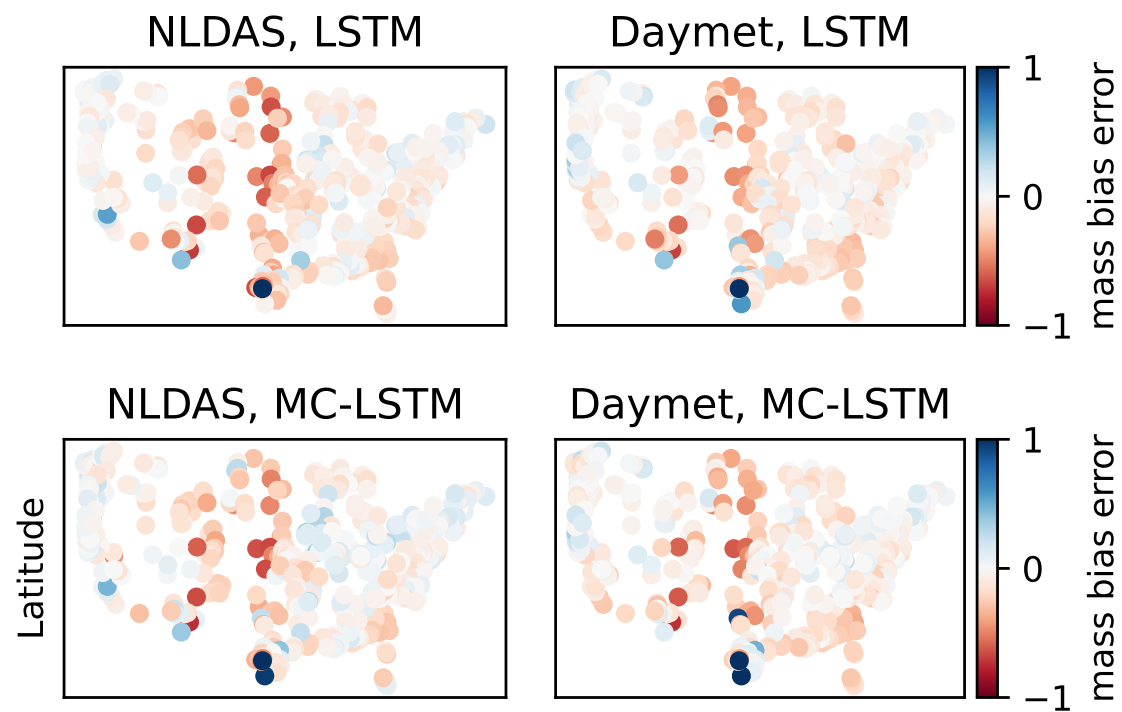

NLDAS, SAC-SMA

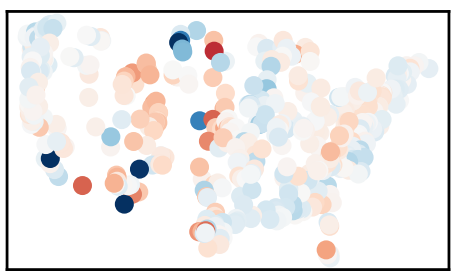

Longitude
Daymet, SAC-SMA

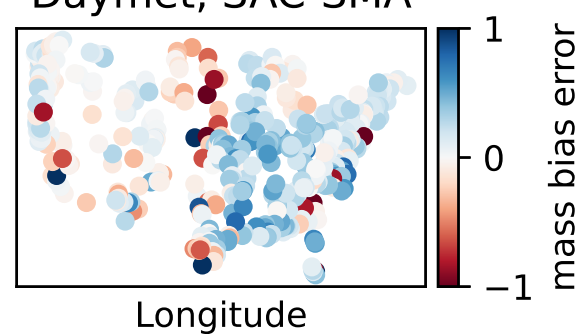

Figure 2. Geospatial distribution of long term positive or negative mass bias error. The left and right columns show the results with NLDAS and Daymet meteorological forcing data, respectively. The three rows are associated (from top to bottom) with LSTM, MC-LSTM and SAC-SMA.

LSTM shows a high mass balance error in the Lower Colorado region, as compared to the MC-LSTM, and the MC-LSTM shows a higher mass balance error in the Rio Grande region, but the LSTM and MC-LSTM are relatively similar (more or less) in the other regions. All three models show relatively high mass bias errors in the CenCon region, which is the contribution from negative mass bias shown in Figure 2. SAC-SMA performs worse than the LSTM and the MC-LSTM in the CenCon region with Daymet forcings.

Figure 4 shows the mass bias errors for the model runs with NLDAS forcings in box and whisker plots for the U.S. Water Resources Regions. With NLDAS forcing, SAC-SMA does not have a consistent mass bias error, as with Daymet. The pattern of SAC-SMA mass bias error in the western U.S. is generally similar between Daymet (Figure 3) and NLDAS (Figure 4). The differences between the LSTM, MC-LSTM and SAC-SMA does not show any obvious patterns. SAC-SMA and LSTM shows a high mass bias error outlier in the Lower Colorado region, but MC-LSTM does not. All three models show relatively high mass bias errors in the CenCon region, although SAC-SMA has a lower mean mass bias error than the LSTM and the MC-LSTM, but has a higher outlier in Missouri. ? shows in their Figure 4 that the 


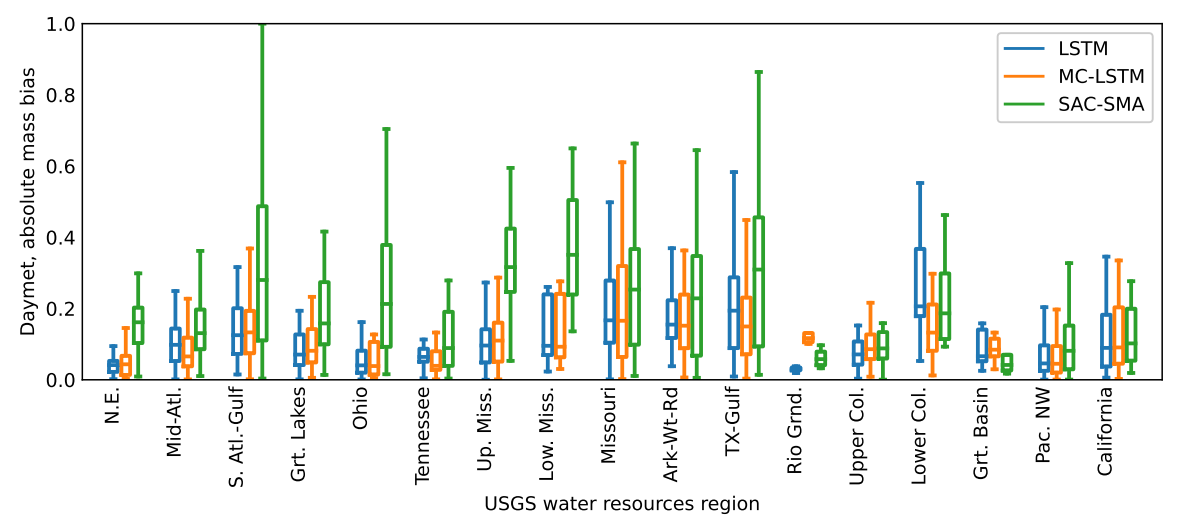

Figure 3. Regional mass balance errors from LSTM, MC-LSTM and SAC-SMA with Daymet forcings. The main body of each box shows the median and confidence intervals. The vertical lines extending to the most extreme, non-outlier data points. Souris-Red-Rainy region (Hydrologic Unit Code 09) is absent due to a lack of sufficient basins.

LSTM scores better in terms of Nash-Sutcliffe Efficiency than SAC-SMA, which seems to indicate that mass bias error in the catchment data does not explain the difference in predictive skill between deep learning and conceptual models.

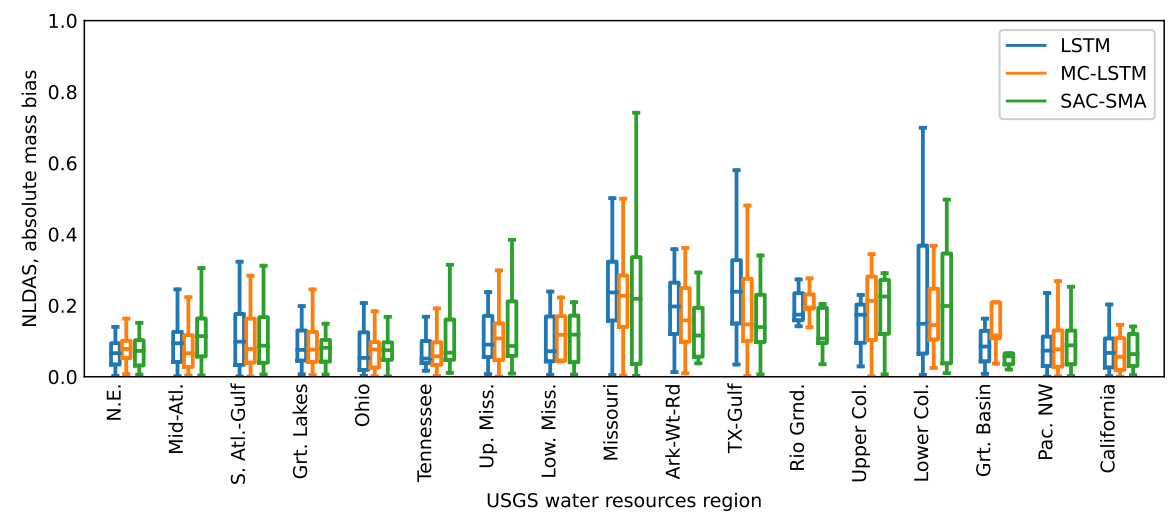

Figure 4. Regional mass balance errors from LSTM, MC-LSTM and SAC-SMA with NLDAS forcings. The main body of each box shows the median and confidence intervals. The vertical lines extending to the most extreme, non-outlier data points. Souris-Red-Rainy region (Hydrologic Unit Code 09) is absent due to a lack of sufficient basins.

Appendix C includes results from a separate time period, where the NWM can be compared (with caveats of the inconsistent calibration period) on the NLDAS forcing data. The overall, spatial and regional results are roughly similar for the LSTM, MC-LSTM and SAC-SMA. 


\subsection{Information loss due to modeling constraints}

\subsubsection{Mutual information}

The mutual information scores of the combined 531 basins (concatenated and calculated once across all basins) with NLDAS forcings are: 0.39 (LSTM), 0.37 (MC-LSTM) and 0.34 (SAC-SMA), respectively. The mutual information scores of the combined 531 basins (concatenated and calculated once across all basins) with Daymet forcings for models LSTM, MC-LSTM and SAC-SMA are 0.40, 0.37 and 0.33, respectively. Figure 5 shows the CDF plots with mutual information scores calculated individually for each basin. For both Daymet and NLDAS the CDF curves show that LSTM has the most mutual information with the observed runoff, followed by MC-LSTM and then by SAC-SMA.
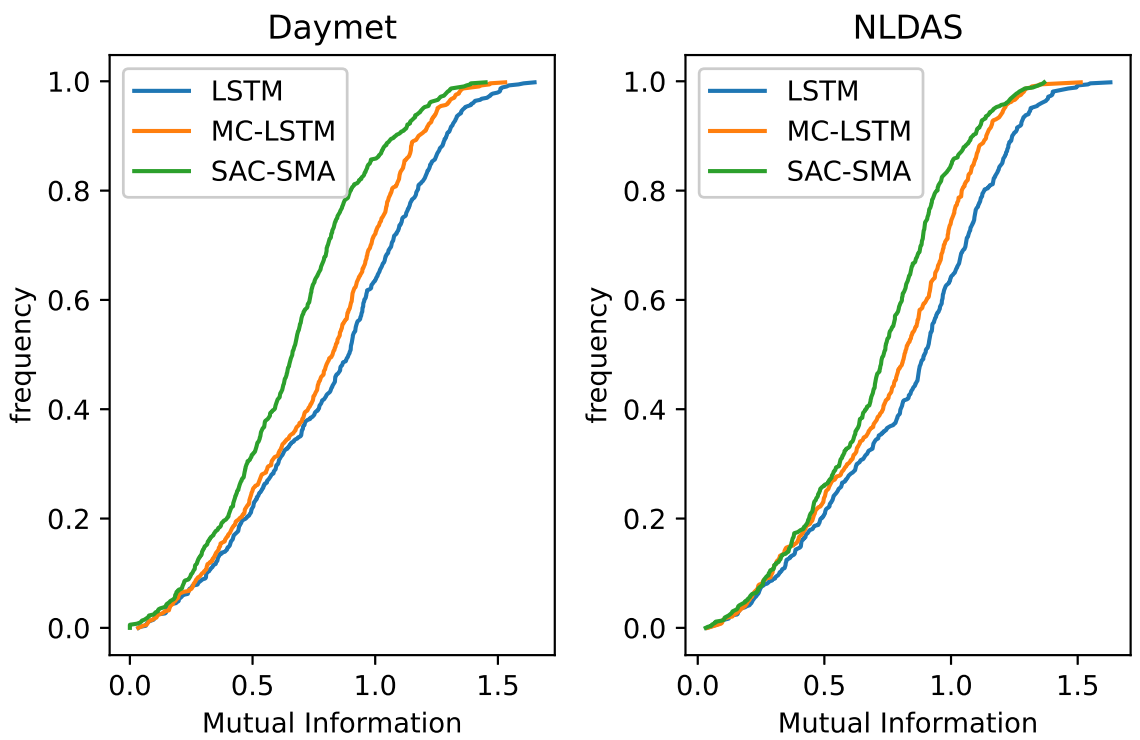

Figure 5. Left: Cumulative distribution of the mutual information for LSTM, MC-LSTM and SAC-SMA with Daymet forcing. Right: Cumulative distribution of the mutual information for LSTM, MC-LSTM and SAC-SMA with NLDAS forcing

\subsubsection{KGE Skill Score}

The unconstrained LSTM was used as a baseline model to measure information loss in the MC-LSTM and SAC-SMA. Results of the KGE skill score $\left(K G E_{s s}\right)$ analysis are shown in Figure 6. The left subplot of this figure shows a clear ordering of model performance that agrees with what we hypothesized in Equation/Inequality 6 - generally, model performance degrades as more constraints are added. The left subplot also shows that DL models perform better when trained and forced with Daymet data than with NLDAS data. This is somewhat counterintuitive given the large, nonstationary bias that we saw in the previous section (Figure 1). SAC-SMA, however, performed significantly worse with the biased data. While the DL models (even those constrained to conserve mass) were able to learn to accommodate the spatially heterogeneous biases in the input data, the PB model was not, even when trained on the biased data in each individual catchment. Daymet is 
the more informative precipitation product overall and a flexible DL model is able to learn and extract this information while the PB model cannot (even though the PB model is locally calibrated), however it is not the mass balance constraints that cause the problem.

The right subplot of Figure 6 plots the CDF of the skill scores (Equation 8) of the MC-LSTM and SAC-SMA relative to the unconstrained LSTM. The gray dotted vertical line represents a skill score of zero, indicating that the test model (MC-LSTM, SAC-SMA) performs equally well as the baseline (LSTM). The main takeaway from this figure is that adding mass balance constraints (both in the MC-LSTM and in SAC-SMA) helps more when using the NLDAS data, even though it was the Daymet data that showed biases.
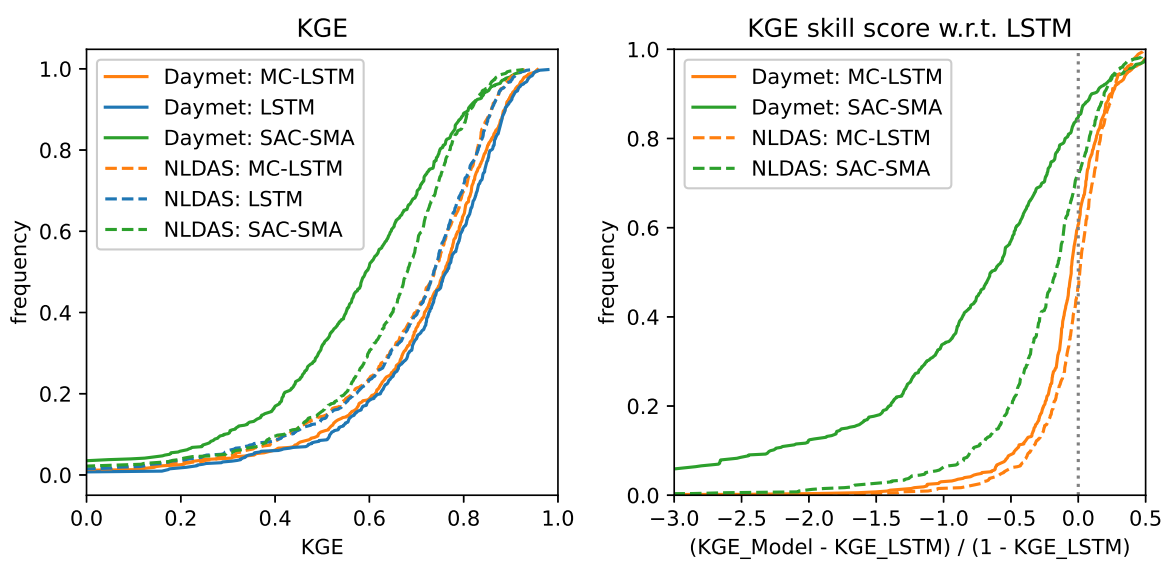

Figure 6. Left: Cumulative distribution of the Kling-Gupta Efficiency (KGE) for three models on two different forcing products. This subplot shows (i) that in general Daymet is more informative than NLDAS, and (ii) that the ordering of the inequality in Equation 6 is generally correct. Right: KGE skill scores (Equation 8) of SAC-SMA and MC-LSTM with respect to the unconstrained LSTM (positive values to the right of the dotted gray line mean that the MC-LSTM(SAC-SMA) performs better than the LSTM, and negative values to the left of the gray line mean that the MC-LSTM(SAC-SMA) performs worse than the LSTM). This subplot shows that adding mass balance constraints to the LSTM has more benefit when using NLDAS inputs than when using Daymet inputs.

Figure 7 plots the CDF of the difference between the MC-LSTM and the LSTM. In each basin, this difference represents an upper bound on the error introduced by mass balance constraints, relative to the LSTM. There are other possible reasons why the MC-LSTM might not perform as well as the LSTM (e.g., the way that it handles unobserved sources and sinks), however this difference (which is sometimes negative) is a conservative estimate of the error due to mass conservation in DL rainfall runoff models.

\section{Conclusions}

275 The hypothesis tested in this paper is that errors in input/output (precipitation/streamflow) data cause apparent violations of closure that may largely explain the poor performance of conceptual and process-based models relative to deep learning. Given that the physical principle of mass balance over a control volume is one of the most fundamental components of hydrological theory, and is the first assumption we take for granted when developing theory-based hydrological models, it is arguably the first physical "law" that we might test when developing physics-informed ML strategies for hydrology. 


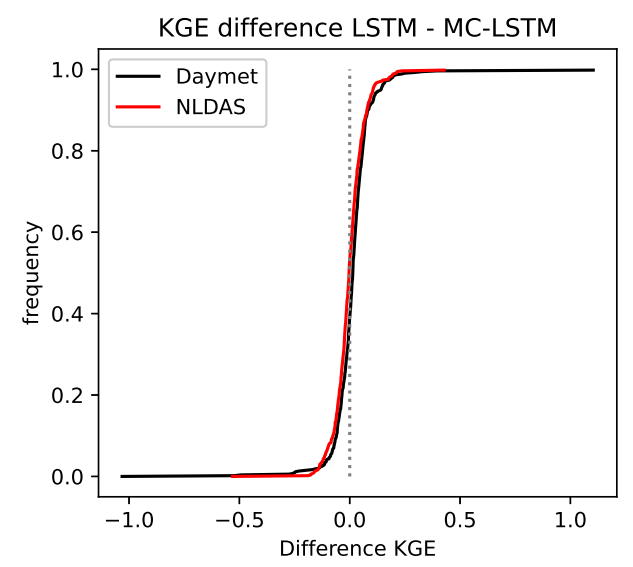

Figure 7. CDF of an estimated upper bound on the error introduced into DL streamflow predictions by adding a mass balance constraint.

For Beven's closure-violation hypothesis to be true as an explanation for the general failure of traditional hydrology models, the errors in rainfall or discharge data must necessarily be systematic in a way that can be learned by a neural network, but not by a calibrated conceptual model. Our results indicate that this is indeed the case: the DL-based LSTM network was able to learn the non-uniform patterns of biases in input-output data, and thereby extract useful information from different (imperfect) precipitation products in spatially and temporally heterogeneous ways. This was true even when using heavily biased rainfall products that contribute significant so-called "disinformation" when calibrating a conceptual model; e.g., Daymet seems to actually contain more information about streamflow than the less biased NLDAS precipitation product. It is important to note that these data biases are not simple additive shifts in the mean - instead they are complex and heterogeneous throughout our large-sample dataset, and the DL models are able largely to learn this heterogeneity.

While Beven (2020) was correct that the imposition of conservation laws is generally harmful for hydrologic prediction, this fact does not help to explain most of the significantly better skill provided by DL over traditional (theory-based) rainfall-runoff models. These findings demonstrate two things:

1. that conservation laws may not be a good foundation for scale-relevant hydrological theory, and

2. that Beven and Westerberg's 2011 claim that disinformative data is a major source of modeling error is — in general — incorrect. In fact, data that are "disinformative" when used in the context of calibrating poorly conceived models might actually contain significant amounts of useful information that is accessible when used in the context of better conceived models. In other words, for catchment-scale rainfall-runoff prediction it is arguably the current hydrological theory that is (more) disinformative, not the hydrological data. In summary, model performance degrades as constraints are added, which causes loss of information between the inputs (atmospheric forcings) and the target (streamflow), as shown in 2.3.3.

\section{Discussion}

There is some subtlety to this conclusion due to the fact that the MC-LSTM includes a flux term that accounts for unobserved sources and sinks (e.g., evapotranspiration, sublimation, aquifer recharge). However, it is important to note that most or all hydrology models that are 
based on closure equations include a residual term in some form. Like all mass balance models, the MC-LSTM explicitly accounts for all water in and across the boundaries of the system. In the case of the MC-LSTM, this residual term is a single aggregated flux. Even with this strong constraint, the MC-LSTM performs significantly better than the mass-conserving benchmark conceptual model. This result indicates that classical hydrology model structures (conceptual architectures and flux equations) actually cause prediction errors that are larger than can be explained as being due to errors in the forcing and observation data.

Our ability to properly conduct a more rigorous and detailed analysis of long-term water balances is limited by the fact that accurate evapotranspiration and percolation data (etc.) are not readily available at watershed scales. Nonetheless, what our analysis based on examining cumulative discharge shows is that an LSTM architecture not constrained to conserve mass is able to extract information from the available data that enables it to learn "effective" water balances that are similar to those learned by a similar model architecture (MC-LSTM) that is explicitly constrained to enforce such closure, and that this effective water balance is in general better than that achieved by traditional conceptual and PB model architectures.

A likely reason for this is that that the current body of hydrological theory does not aggregate well to the scale of unorganized complex watershed systems (Nearing et al., 2020c). While it is true that hydrological theory can enable a modeler to "interpret" a watershed response (assuming a proper accounting for uncertainty), such theory does not currently translate into accurate predictions of catchment-scale behaviors using available data. Meanwhile, the most accurate way to generate a predictive model is to impose as few "physical constraints" as possible on its ability to extract information from the available data, and consequently any model that is constrained to obey some "deeper" physical understanding of the system must be less accurate in a predictive sense, unless that physical understanding actually contributes predictively-useful information that cannot be otherwise extracted directly from the data.

Looking forward, a particular application of rainfall-runoff modeling that necessarily requires the imposition of strict mass-balance constraints is "Earth-system-scale" modeling. In this context, any model that seeks to explain components of long-term climate variability (for instance) cannot allow for any significant amount of residual mass to go unexplained. To use a dramatic example, unaccounted for losses at the catchment-scale could potentially result in the removal of all water mass from the global water cycle, which would render a long-term simulation useless. Global-scale modeling of land-surface dynamics could be a potentially powerful application of the MC-LSTM network approach, but such an implementation would require partitioning the single mass-loss representation (referred to above as the "trash-bucket") between "losses" (transfers) to the sub-surface and "losses" (transfers) to the atmosphere. 
Code and data availability. All LSTMs and MC-LSTMs were trained using the NeuralHydrology Python library available at https://github. com/neuralhydrology/neuralhydrology. A snapshot of the exact version that we used is available at https:/github.com/jmframe/mclstm 2021_extrapolate/neuralhydrology and under DOI number 10.5281/zenodo.5051961. Code for calibrating SAC-SMA is from https://github. com/Upstream-Tech/SACSMA-SNOW17, which includes the SpotPy calibration library https://pypi.org/project/spotpy/. Input data for all model runs except the NWM-Rv2 came from the public NCAR CAMLES repository https://ral.ucar.edu/solutions/products/camels and were used according to instructions outlined in the NeuralHydrology readme. NWM-Rv2 data are available publicly from https://registry. opendata.aws/nwm-archive/. All model output data generated by this project will be available on the CUAHSI HydroShare platform under a DOI number https://doi.org/10.4211/hs.d750278db868447dbd252a8c5431affd. Interactive Python scripts for all post-hoc analysis reported in this paper, including calculating metrics and generating tables and figures, are available at https://github.com/jmframe/mclstm_2021_ mass_balance

Author contributions. Jonathan Frame conceived the experimental design, performed all analysis of experiments and wrote the manuscript. Frederik Kratzert wrote the LSTM and MC-LSTM code. Hoshin Gupta and Paul Ullrich advised the manuscript and participated in interpretation of results. Grey Nearing advised on experimental design, model training/calibration, development of the MC-LSTM and contributed the manuscript.

Competing interests. The authors report no competing interests. 


\section{References}

Addor, N., Newman, A. J., Mizukami, N., and Clark, M. P.: The CAMELS data set: catchment attributes and meteorology for large-sample studies, Hydrology and Earth System Sciences (HESS), 21, 5293-5313, 2017.

Bennett, A. and Nijssen, B.: Deep Learned Process Parameterizations Provide Better Representations of Turbulent Heat Fluxes in Hydrologic Models, Water Resources Research, 57, 1-14, https://doi.org/10.1029/2020WR029328, 2021.

Beven, K.: Deep learning, hydrological processes and the uniqueness of place, Hydrological Processes, 34, 3608-3613, 2020.

Beven, K. and Westerberg, I.: On red herrings and real herrings: Disinformation and information in hydrological inference, Hydrological Processes, 25, 1676-1680, https://doi.org/10.1002/hyp.7963, 2011.

Beven, K. J.: Uniqueness of place and process representations in hydrological modelling, https://doi.org/10.5194/hess-4-203-2000, 2000.

Beven, K. J., Smith, P. J., and Freer, J. E.: So just why would a modeller choose to be incoherent?, Journal of Hydrology, 354, 15-32, https://doi.org/10.1016/j.jhydrol.2008.02.007, 2008.

Chow, V. T., Maidment, D. R., and Mays, L. W.: Applied Hydrology Chow 1988.pdf, McGraw-Hill, http://ponce.sdsu.edu/Applied_ Hydrology_Chow_1988.pdf, 1988.

Cover, T. M. and Thomas, J. A.: Elements of Information Theory, https://doi.org/10.1002/047174882X, 2005.

Daw, A., Thomas, R. Q., Carey, C. C., Read, J. S., Appling, A. P., and Karpatne, A.: Physics-Guided Architecture (PGA) of Neural Networks for Quantifying Uncertainty in Lake Temperature Modeling, Proceedings of the 2020 SIAM International Conference on Data Mining, pp. 532-540, https://doi.org/10.1137/1.9781611976236.60, 2020.

Frame, J. M., Kratzert, F., Klotz, D., Gauch, M., Shalev, G., Gilon, O., Qualls, L. M., Gupta, H. V., and Nearing, G. S.: Deep learning rainfall-runoff predictions of extreme events, pp. 1-20, 2021a.

Frame, J. M., Raney, A., Salas, F., Kratzert, F., Rahman, M., and Nearing, G.: Post processing the US National Water Model with a Long Short-Term Memory network, Journal of the American Water Research Association, 2021b.

Gauch, M., Kratzert, F., Klotz, D., Nearing, G., Lin, J., and Hochreiter, S.: Rainfall-runoff prediction at multiple timescales with a single Long Short-Term Memory network, Hydrology and Earth System Sciences, 25, 2045-2062, $2021 \mathrm{a}$.

Gauch, M., Mai, J., and Lin, J.: The proper care and feeding of CAMELS: How limited training data affects streamflow prediction, Environmental Modelling \& Software, 135, 104 926, 2021b.

Gupta, H. V. and Nearing, G. S.: Debates-The future of hydrological sciences: A (common) path forward? Using models and data to learn: A systems theoretic perspective on the future of hydrological science, Water Resources Research, 50, 5351-5359, 2014.

Gupta, H. V., Kling, H., Yilmaz, K. K., and Martinez, G. F.: Decomposition of the mean squared error and NSE performance criteria: Implications for improving hydrological modelling, Journal of hydrology, 377, 80-91, 2009.

Hochreiter, S. and Schmidhuber, J.: Long short-term memory, Neural computation, 9, 1735-1780, 1997.

Hoedt, P.-J., Kratzert, F., Klotz, D., Halmich, C., Holzleitner, M., Nearing, G. S., Hochreiter, S., and Klambauer, G.: MC-LSTM: MassConserving LSTM, in: Proceedings of the 38th International Conference on Machine Learning, edited by Meila, M. and Zhang, T., vol. 139 of Proceedings of Machine Learning Research, pp. 4275-4286, PMLR, http://proceedings.mlr.press/v139/hoedt21a.html, 2021.

Houska, T., Kraft, P., Chamorro-Chavez, A., and Breuer, L.: SPOTPY: A Python library for the calibration, sensitivity-and uncertainty analysis of Earth System Models., in: Geophysical Research Abstracts, vol. 21, 2019.

Jia, X., Willard, J., Karpatne, A., Read, J. S., Zwart, J. A., Steinbach, M., and Kumar, V.: Physics-Guided Machine Learning for Scientific Discovery: An Application in Simulating Lake Temperature Profiles, pp. 1-25, http://arxiv.org/abs/2001.11086, 2020. 
Jiang, S., Zheng, Y., and Solomatine, D.: Improving AI system awareness of geoscience knowledge: Symbiotic integration of physical approaches and deep learning, Geophysical Research Letters, 47, e2020GL088 229, 2020.

Karniadakis, G. E., Kevrekidis, I. G., Lu, L., Perdikaris, P., Wang, S., and Yang, L.: Physics-informed machine learning, Nature Reviews Physics, https://doi.org/10.1038/s42254-021-00314-5, 2021.

Klotz, D., Kratzert, F., Gauch, M., Keefe Sampson, A., Brandstetter, J., Klambauer, G., Hochreiter, S., and Nearing, G.: Uncertainty Estimation with Deep Learning for Rainfall-Runoff Modelling, Hydrology and Earth System Sciences Discussions, pp. 1-32, 2021.

Knoben, W. J. M., Freer, J. E., and Woods, R. A.: Technical note : Inherent benchmark or not ? Comparing Nash - Sutcliffe and Kling Gupta efficiency scores, pp. 4323-4331, 2019.

Kratzert, F., Klotz, D., Brenner, C., Schulz, K., and Herrnegger, M.: Rainfall-runoff modelling using long short-term memory (LSTM) networks, Hydrology and Earth System Sciences, 22, 6005-6022, 2018.

Kratzert, F., Klotz, D., Herrnegger, M., Sampson, A. K., Hochreiter, S., and Nearing, G. S.: Toward Improved Predictions in Ungauged Basins: Exploiting the Power of Machine Learning, Water Resources Research, 55, 11344-11354, https://doi.org/https://doi.org/10.1029/2019WR026065, 2019a.

Kratzert, F., Klotz, D., Shalev, G., Klambauer, G., Hochreiter, S., and Nearing, G.: Towards learning universal, regional, and local hydrological behaviors via machine learning applied to large-sample datasets, Hydrology and Earth System Sciences, 23, 5089-5110, https://doi.org/10.5194/hess-23-5089-2019, 2019b.

Kratzert, F., Klotz, D., Hochreiter, S., and Nearing, G. S.: A note on leveraging synergy in multiple meteorological data sets with deep learning for rainfall-runoff modeling, Hydrology and Earth System Sciences, 25, 2685-2703, https://doi.org/10.5194/hess-25-2685-2021, 2021.

Nash, J. E. and Sutcliffe, J. V.: River flow forecasting through conceptual models part I-A discussion of principles, Journal of hydrology, 10, 282-290, 1970.

Nearing, G., Research, G., Kratzert, F., Klotz, D., Hoedt, P.-J., Klambauer, G., Hochreiter, S., Gupta, H., Nevo, S., and Matias, Y.: A Deep Learning Architecture for Conservative Dynamical Systems: Application to Rainfall-Runoff Modeling, AI for Earth Sciences Workshop at NEURIPS 2020, 2020a.

Nearing, G., Sampson, A. K., Kratzert, F., and Frame, J.: Post-processing a Conceptual Rainfall-runoff Model with an LSTM, 2020b.

Nearing, G. S. and Gupta, H. V.: The quantity and quality of information in hydrologic models, Water Resources Research, 51, 524-538, 2015.

Nearing, G. S., Kratzert, F., Sampson, A. K., Pelissier, C. S., Klotz, D., Frame, J. M., Prieto, C., and Gupta, H. V.: What role does hydrological science play in the age of machine learning?, Water Resources Research, p. e2020WR028091, 2020c.

Newman, A., Clark, M., Sampson, K., Wood, A., Hay, L., Bock, A., Viger, R., Blodgett, D., Brekke, L., Arnold, J., et al.: Development of a large-sample watershed-scale hydrometeorological data set for the contiguous USA: data set characteristics and assessment of regional variability in hydrologic model performance, Hydrology and Earth System Sciences, 19, 209, 2015.

Newman, A. J., Mizukami, N., Clark, M. P., Wood, A. W., Nijssen, B., and Nearing, G.: Benchmarking of a physically based hydrologic model, Journal of Hydrometeorology, 18, 2215-2225, 2017.

Niu, G.-Y., Yang, Z.-L., Mitchell, K. E., Chen, F., Ek, M. B., Barlage, M., Kumar, A., Manning, K., Niyogi, D., Rosero, E., et al.: The community Noah land surface model with multiparameterization options (Noah-MP): 1. Model description and evaluation with localscale measurements, Journal of Geophysical Research: Atmospheres, 116, 2011. 
Pelissier, C., Frame, J., and Nearing, G.: Combining Parametric Land Surface Models with Machine Learning, http://arxiv.org/abs/2002. 06141, 2019.

Reichstein, M., Camps-Valls, G., Stevens, B., Jung, M., Denzler, J., Carvalhais, N., et al.: Deep learning and process understanding for data-driven Earth system science, Nature, 566, 195-204, 2019.

Salas, F. R., Somos-Valenzuela, M. A., Dugger, A., Maidment, D. R., Gochis, D. J., David, C. H., Yu, W., Ding, D., Clark, E. P., and Noman, N.: Towards real-time continental scale streamflow simulation in continuous and discrete space, JAWRA Journal of the American Water Resources Association, 54, 7-27, 2018.

Shen, C., Chen, X., and Laloy, E.: Editorial: Broadening the Use of Machine Learning in Hydrology, Frontiers in Water, 3, 1-4, https://doi.org/10.3389/frwa.2021.681023, 2021.

Sivapalan, M., Takeuchi, K., Franks, S. W., Gupta, V. K., Karambiri, H., Lakshmi, V., Liang, X., McDonnell, J. J., Mendiondo, E. M., O'Connell, P. E., Oki, T., Pomeroy, J. W., Schertzer, D., Uhlenbrook, S., and Zehe, E.: IAHS Decade on Predictions in Ungauged Basins (PUB), 2003-2012: Shaping an exciting future for the hydrological sciences, Hydrological Sciences Journal, 48, 857-880, https://doi.org/10.1623/hysj.48.6.857.51421, 2003.

Tsai, W.-P., Pan, M., Lawson, K., Liu, J., Feng, D., and Shen, C.: From parameter calibration to parameter learning: Revolutionizing largescale geoscientific modeling with big data, arXiv preprint arXiv:2007.15751, 2020.

USGS: Hydrologic Unit Maps: U.S. Geological Survey Water-Supply Paper 2294, http://pubs.usgs.gov/wsp/wsp2294/pdf/wsp\{_\}2294.pdf, 1987.

Willard, J., Jia, X., Xu, S., Steinbach, M., and Kumar, V.: Integrating Scientific Knowledge with Machine Learning for Engineering and Environmental Systems, 1, 1-35, http://arxiv.org/abs/2003.04919, 2021.

435 Xie, K., Liu, P., Zhang, J., Han, D., Wang, G., and Shen, C.: Physics-guided deep learning for rainfall-runoff modeling by considering extreme events and monotonic relationships, Journal of Hydrology, 603, 127 043, https://doi.org/10.1016/j.jhydrol.2021.127043, 2021.

Zhao, W. L., Gentine, P., Reichstein, M., Zhang, Y., Zhou, S., Wen, Y., Lin, C., Li, X., and Qiu, G. Y.: Physics-Constrained Machine Learning of Evapotranspiration, Geophysical Research Letters, 46, 14 496-14 507, https://doi.org/10.1029/2019GL085291, 2019. 


\section{Appendix A: LSTM}

Long Short Term Memory networks (Hochreiter and Schmidhuber, 1997) are dynamic state-space recurrent neural networks that are well known to be appropriate for rainfall-runoff modeling (Kratzert et al., 2018). The rainfall-runoff process is a time evolving system that is comprised of 1) the state of the watershed, and 2) the dynamic response to inputs. This is the same general principal on which recurrent neural networks operate.

The input to an LSTM, as we use in hydrologic modeling, is a vector of both forcings (values that change with time) and static attributes of the particular system under consideration. Our LSTM is run at a discrete timestep, in this case a daily timestep, making the input vector, $\boldsymbol{x}[t]$, with forcings representing the daily values. This LSTM is trained to represent the dynamics between the input-state-output relationship at this particular timestep. Given a time sequence of inputs $(\boldsymbol{x}=[\boldsymbol{x}[1], \ldots, \boldsymbol{x}[T]])$, which act on the state of the previous timestep $(\boldsymbol{h}[t-1])$ and we get an output sequence $(\boldsymbol{y}=[\boldsymbol{y}[1], \ldots, \boldsymbol{y}[T]])$.

The LSTM network as a discrete timestepping model is represented as the following equations:

$\boldsymbol{i}[t]=\sigma\left(\boldsymbol{W}_{\boldsymbol{i}} \boldsymbol{x}[t]+\boldsymbol{U}_{\boldsymbol{i}} \boldsymbol{h}[t-1]+\boldsymbol{b}_{\boldsymbol{i}}\right)$

$\boldsymbol{f}[t]=\sigma\left(\boldsymbol{W}_{\boldsymbol{f}} \boldsymbol{x}[t]+\boldsymbol{U}_{\boldsymbol{f}} \boldsymbol{h}[t-1]+\boldsymbol{b}_{\boldsymbol{f}}\right)$

$\boldsymbol{g}[t]=\tanh \left(\boldsymbol{W}_{\boldsymbol{g}} \boldsymbol{x}[t]+\boldsymbol{U}_{\boldsymbol{g}} \boldsymbol{h}[t-1]+\boldsymbol{b}_{\boldsymbol{g}}\right)$

$\boldsymbol{o}[t]=\sigma\left(\boldsymbol{W}_{o} \boldsymbol{x}[t]+\boldsymbol{U}_{\boldsymbol{o}} \boldsymbol{h}[t-1]+\boldsymbol{b}_{\boldsymbol{o}}\right)$

$\boldsymbol{c}[t]=\boldsymbol{f}[t] \odot \boldsymbol{c}[t-1]+\boldsymbol{i}[t] \odot \boldsymbol{g}[t]$

$\boldsymbol{h}[t]=\boldsymbol{o}[t] \odot \tanh (\boldsymbol{c}[t])$,

The symbols $\boldsymbol{i}[t], \boldsymbol{f}[t]$ and $\boldsymbol{o}[t]$ refer to the input gate, forget gate, and output gate of the LSTM respectively, $\boldsymbol{g}[t]$ is the cell input and $\boldsymbol{x}[t]$ is the network input at time step $t, \boldsymbol{h}[t-1]$ is the LSTM output, which is also called the recurrent input because it is used as inputs to all gates in the next timestep, and $c[t-1]$ is the cell state from the previous time step.

Cell states contain information of the system at any point in the discrete time. $\sigma(\cdot)$ are sigmoid activation functions, which return values in $[0,1]$, which act as an attention of the internal network dynamics. The forget gate controls the memory timescales of each of the cell states, and the input and output gates control flows of information from the input features to the cell states and from the cell states to the outputs (recurrent inputs), respectively. $\boldsymbol{W}, \boldsymbol{U}$ and $\boldsymbol{b}$ are parameters that are tuned such that the dynamics of the LSTM produce an output that matches the target output, streamflow in our case. Parameter subscripts $(i, f, g \& o)$ indicate which gate the particular parameter matrix/vector is associated with. $\tanh (\cdot)$ is the hyperbolic tangent activation function, which serves to add nonlinearity to the model in the cell input and recurrent input, and $\odot$ indicates element-wise multiplication. For a hydrological interpretation of the LSTM, see Kratzert et al. (2018).

\section{Appendix B: Mass conserving LSTM}

The LSTM can be trained to represent system dynamics, but without constraints that are typically used to represent a physical system. Although similar to the type of models that use a physical conceptualization to represent system dynamics, the LSTM lacks a few key ingrediants, including physical units associate with the system inputs and outputs. The MC-LSTM aims to bridge the gap by enforcing 1 within the LSTM arcitecture. Using the notation from Appendix A, this is:

$\boldsymbol{c}^{*}[t]=\boldsymbol{c}^{*}[t-1]+\boldsymbol{x}^{*}[t]-\boldsymbol{h}^{*}[t]$, 


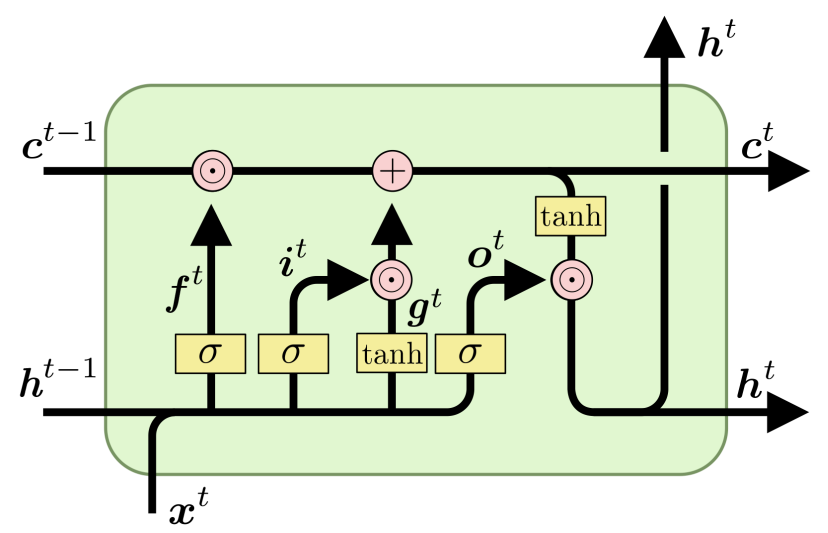

Figure A1. A single timestep of a standard LSTM with timesteps marked as superscripts for clarity. $\boldsymbol{x}^{t}, \boldsymbol{c}^{t}$, and $\boldsymbol{h}^{t}$ are the input features, cell states, and recurrent inputs at time $t$, respectively. $\boldsymbol{f}^{t}, \boldsymbol{i}^{t}$, and $\boldsymbol{o}^{t}$ are the forget-, input- and output-gate and $\boldsymbol{g}^{t}$ denotes the cell input. Boxes labeled $\sigma$ and tanh represent single sigmoid and hyperbolic tangent activation layers with the same number of nodes as cell states. The addition sign represent element-wise addition and $\odot$ represents element-wise multiplication.

where $\boldsymbol{c}^{*}[t], \boldsymbol{x}^{*}[t]$ and $\boldsymbol{h}^{*}[t]$ are components of the cell states, input features, and model outputs (recurrent inputs) that contribute to a particular conservation law.

Two major modifications to the LSTM are required to enforce mass conservation, 1) activation functions that ensure of all elements sum to one (through normalization) on gates where mass passes, and 2) subtracting the mass out of the system from the cell states (1) (Hoedt et al., 2021).

The constrained model architecture is illustrated in Fig. B1. Note that that the inputs are separated into mass inputs $\boldsymbol{x}$ and auxiliary inputs $a$. In our case, the mass input is precipitation and the auxiliary inputs are everything else (e.g. temperature, radiation, catchment attributes). The input gate (sigmoids) and cell input (hyperbolic tangents) in the standard LSTM are (collectively) replaced by one of these normalization layers, while the output gate is a standard sigmoid gate, similar to the standard LSTM. The forget gate is also replaced by a normalization layer, with the important difference that the output of this layer is a square matrix with dimension equal to the size of the cell state. This matrix is used to "reshuffle" the mass between the cell states at each timestep. This reshuffling matrix is column-wise normalized so that the dot product with the cell state vector at time $t$ results in a new cell state vector having the same absolute norm (so that no mass is lost or gained). 
We call this general architecture a Mass-Conserving LSTM (MC-LSTM), even though it works for any type of conservation law (mass, energy, momentum, counts, etc.). The architecture is illustrated in Figure B1 and is described formally as follows:

$$
\begin{aligned}
\hat{\boldsymbol{c}}[t-1] & =\frac{\boldsymbol{c}[t-1]}{\|\boldsymbol{c}[t-1]\|_{1}} \\
\boldsymbol{i}[t] & =\widehat{\sigma}\left(\boldsymbol{W}_{i} \boldsymbol{x}[t]+\boldsymbol{U}_{i} \hat{\boldsymbol{c}}[t-1]+\boldsymbol{V}_{i} \boldsymbol{a}[t]+\boldsymbol{b}_{i}\right) \\
\boldsymbol{o}[t] & =\sigma\left(\boldsymbol{W}_{o} \boldsymbol{x}[t]+\boldsymbol{U}_{o} \hat{\boldsymbol{c}}[t-1]+\boldsymbol{V}_{o} \boldsymbol{a}[t]+\boldsymbol{b}_{\boldsymbol{o}}\right) \\
\boldsymbol{R}[t] & =\widehat{\operatorname{ReLU}}\left(\mathbf{W}_{R} \boldsymbol{x}[t]+\mathbf{U}_{R} \hat{\boldsymbol{c}}[t-1]+\mathbf{V}_{R} \boldsymbol{a}[t]+\boldsymbol{b}_{R}\right) \\
\boldsymbol{m}[t] & =\boldsymbol{R}[t] \boldsymbol{c}[t-1]+\boldsymbol{i}[t] \boldsymbol{x}[t] \\
\boldsymbol{c}[t] & =(1-\boldsymbol{o}[t]) \odot \boldsymbol{m}[t] \\
\boldsymbol{h}[t] & =\boldsymbol{o}[t] \odot \boldsymbol{m}[t]
\end{aligned}
$$

Learned parameters are $\boldsymbol{W}, \boldsymbol{U}, \boldsymbol{V}$, and $\boldsymbol{b}$ for all of the gates. The normalized activation functions are, in this case, $\widehat{\sigma}\left(\frac{\sigma\left(s_{k}\right)}{\sum_{k} \sigma\left(s_{k}\right)}\right)$ for the input gate and $\widehat{\operatorname{ReLU}}\left(\frac{\max \left(s_{k}, 0\right)}{\sum_{k} \max \left(s_{k}, 0\right)}\right)$ for the redistribution matrix $\boldsymbol{R}$, as in the hydrology example of Hoedt et al. (2021). The product of $\boldsymbol{i}[t] \boldsymbol{x}[t]$ and $\boldsymbol{o}[t] \odot \boldsymbol{m}[t]$ are input and output fluxes, respectively.

Because this model structure is fundamentally conservative, all cell states and information transfers within the model are associated with physical units. Our objective in this study was to maintain the overall water balance in a catchment - our conserved input feature, $\boldsymbol{x}$, is precipitation in units $[\mathrm{mm} / \mathrm{day}]$ and our training targets are catchment discharge also in units of $[\mathrm{mm} / d a y]$. Thus, all input fluxes, output fluxes, and cell states in the MC-LSTM have units of $[\mathrm{mm} / \mathrm{day}]$.

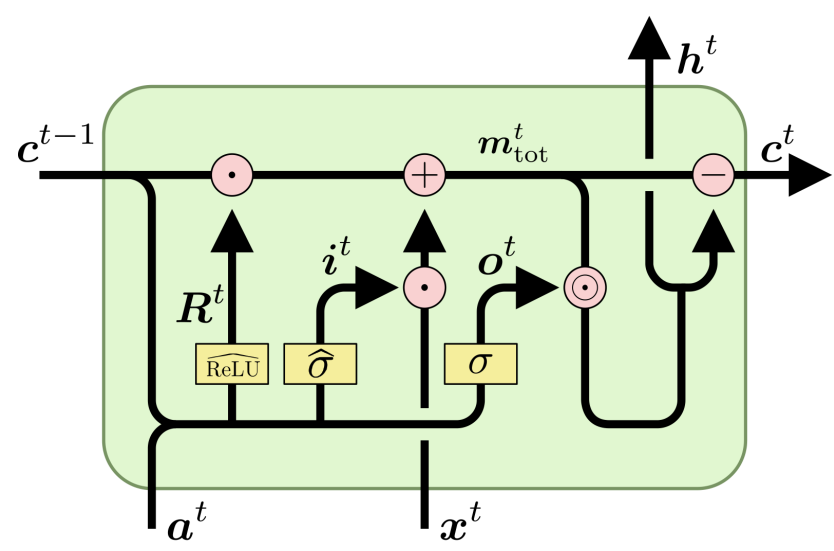

Figure B1. A single timestep of a Mass-Conserving LSTM with timesteps marked as superscripts for clarity. As in Figure A1, $\boldsymbol{c}^{t}, \boldsymbol{a}^{t}, \boldsymbol{x}^{t}$, $\boldsymbol{i}^{t}, \boldsymbol{o}^{t}$, and $\boldsymbol{R}^{t}$ are the cell states, conserved inputs, input features, input fluxes, output fluxes, and reshuffling matrix at time $t$, respectively. $\sigma$ represents a standard sigmoid activation layer, $\widehat{\sigma}$ and $\widehat{\operatorname{ReLU}}$ represent normalized sigmoid activation layers and normalized ReLU activation layer respectively. Addition and subtraction signs represent element-wise addition and subtraction, $\odot$ represents element-wise multiplication and the $\cdot$ sign represents the dot-product. 


\section{Appendix C: Comparison with the U.S. National Water Model}

The process-based model that we used as a benchmark was the NOAA National Water Model (NWM) retrospective run version 2 (NWMRv2). The NWM is based on WRF-Hydro (Salas et al., 2018), which is a process-based model that includes Noah-MP (Niu et al., 2011) as a land surface component, kinematic wave overland flow, and Muskingum-Cunge channel routing. NWM-Rv2 was previously used as a benchmark for LSTM simulations in CAMELS by Kratzert et al. (2019a), Gauch et al. (2021a) and Frame et al. (2021b). Public data from NWM-Rv2 is hourly and CONUS-wide - we pulled hourly flow estimates from the USGS gauges in the CAMELS data set and averaged these hourly data to daily over the time period October 1, 1980 through September 30, 2008. As a point of comparison, Gauch et al. (2021a) compared hourly and daily LSTM predictions against the NWM-Rv2 and found that the NWM-Rv2 was significantly more accurate at the daily timescale than at the hourly timescale, whereas the LSTM did not lose accuracy at the hourly timescale vs. the daily timescale. All experiments in the present study were done at the daily timescale.

The NWM is also susceptible to the kinds of mass bias error propagation from the forcings. We can't, however, test the same hypothesis with the NWM because we do not have the capability to re-calibrate and run the NWM with Daymet forcing. The National Oceanic and Atmospheric Association (NOAA) has made publicly available a NWM retrospective run using NLDAS forcing data. This allows us to directly compare the mass balance errors with the LSTM, MC-LSTM and SAC-SMA. The NWM retrospecive run (NWM-Rv2) does not completely overlap with our test period (1989-1999). We performed the same experiment on a test period that can be compared with the NWM-Rv2, which includes training/calibrated the LSTM, MC-LSTM and SAC-SMA. The train/test period split used a test period that aligns with the availability of benchmark data from the US National Water Model. The train period included water years 1981-1995, and the test period included water years 1996-2014 (i.e., from October 1, 1995 through September 30, 2014). This was the same training period used by Newman et al. (2017) and Kratzert et al. (2019a), but with an extended test period. This train/test split was used because the NWM-Rv2 data record is not long enough to accommodate the train/test split used by previous studies (item above in this list).

The NWM-Rv2 was calibrated by NOAA personnel on about 1400 basins with NLDAS forcing data and includes a regionalization strategy that attempts to use the calibrated parameters across basins not included in the calibration set, however most of the CAMELS basins are included in that calibration set. The NWM-Rv2 calibration time period is on water years 2009-2013. Because of the inconsistencies in the time period and basins included in the calibration, we cannot directly compare the NWM-Rv2 to the other models. But we include the NWM-Rv2 here as an appendix because it is relevant to the hydrologic community to see, even if not directly comparable, the results of a physics-based model.

The information loss between the LSTM model and the constrained MC-LSTM and SAC-SMA models for this test period, shown in Figure $\mathrm{C} 1$ is similar to that of the test period shown in the main text, Figure 6. He we also show the information loss from the process-based NWM, which actually shows more information loss than SAC-SMA. This is likely because the NWM has more constraints, that come in the form of a multi-layered modeling chain. The NWM starts with a land surface model, which causes runoff across a terrain routing model, which is also two-way coupled with the land model, and finally the terrain model feeds into the channel routing model, which provides an estimate of streamflow. There are multiple steps along that modeling chain that cause different amounts of information loss.

Figure C2 shows the cumulative density functions (CDFs) of long-term cumulative discharge from the 484 CAMELS basins from the models during the 1996-2014 test period. Note that we excluded basins that did not have a complete observation time series throughout the entire test period. The LSTM and MC-LSTM both predicted streamflows that result in more accurate long-term cumulative discharge than the calibrated SAC-SMA model. The LSTM and the MC-LSTM performed roughly similarly on both NLDAS and Daymet

// // [Insert Figure C2] // // 
Table C1. Median performance metrics (plus or minus the $95 \%$ confidence interval) across 484 basins calculated on the test period $1996-2014$ with two separate forcing products.

\begin{tabular}{l|rrr|rrrr}
\hline \multirow{2}{*}{ Metric } & \multicolumn{4}{|c|}{ Daymet forcing } & \multicolumn{4}{c}{ NLDAS forcing } \\
\hline NSE & LSTM & MC-LSTM & SAC-SMA & LSTM & MC-LSTM & SAC-SMA & NWM* \\
KGE & $0.74 \pm-0.02$ & $0.74 \pm-0.02$ & $0.59 \pm-0.08$ & $0.71 \pm-0.05$ & $0.72 \pm-0.02$ & $0.63 \pm-0.05$ & $0.63 \pm-0.05$ \\
Pearson-r & $0.78 \pm-0.02$ & $0.77 \pm-0.02$ & $0.56 \pm \mathrm{n} / \mathrm{a}$ & $0.77 \pm-0.02$ & $0.74 \pm-0.02$ & $0.68 \pm-0.02$ & $0.67 \pm-0.05$ \\
Alpha-NSE & $0.88 \pm-0.01$ & $0.88 \pm-0.01$ & $0.81 \pm \mathrm{n} / \mathrm{a}$ & $0.86 \pm-0.01$ & $0.86 \pm-0.01$ & $0.81 \pm-0.01$ & $0.82 \pm-0.01$ \\
Beta-NSE & $0.03 \pm-0.01$ & $0.03 \pm-0.01$ & $0.13 \pm-0.02$ & $0.01 \pm-0.01$ & $-0.01 \pm-0.01$ & $-0.01 \pm \mathrm{n} / \mathrm{a}$ & $-0.01 \pm \mathrm{n} / \mathrm{a}$ \\
Peak-Timing & $0.34 \pm-0.03$ & $0.33 \pm-0.03$ & $0.45 \pm-0.06$ & $0.38 \pm-0.03$ & $0.4 \pm-0.03$ & $0.53 \pm-0.06$ & $0.54 \pm-0.05$ \\
\hline
\end{tabular}
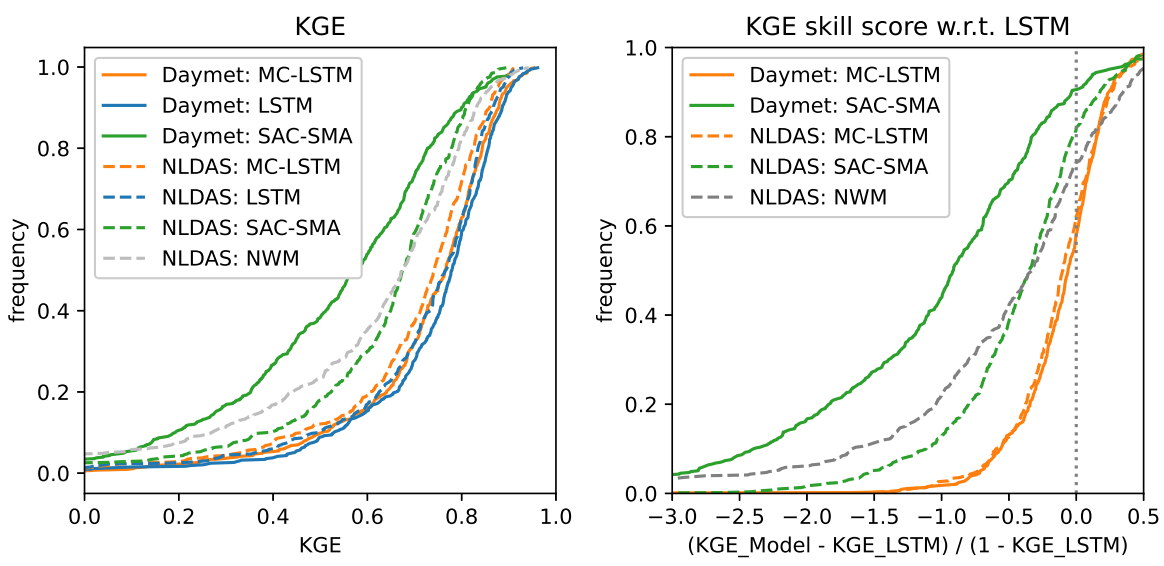

Figure C1. Left: Cumulative distribution of the Kling-Gupta Efficiency (KGE) for four models on two different forcing products. Right: KGE skill score with respect to the unconstrained LSTM.

Figure C3 shows the Mass balance results from for the three models with both Daymet and NLDAS forcings. The result of the SAC-SMA simulation with Daymet forcings shows a clear positive mass bias error in the eastern half of CONUS. The result of the simulation with NLDAS forcings shows a mix of positive and negative mass bias throughout CONUS.

Figure C4 shows the mass bias errors for the model runs with NLDAS forcings in box and whisker plots for the U.S. Water Resources Regions. A mass bias error is clearly shows for SAC-SMA in the easter CONUS regions, while the LSTM and MC-LSTM do not express this pattern. There is generally a correlation between the three models, where the regions with high mass bias error are expressed by all three models. For instance, the Upper Colorado region shows low mass bias error for all three models, and the Lower Colorado shows a relatively high mass bias error by all three models.

Figure C5 shows the mass bias errors for the model runs with Daymet forcings in box and whisker plots for the U.S. Water Resources Regions. The NWM-Rv2 has high outliers in the Central CONUS regions. There is a correlation of mass bias error across all four models, 

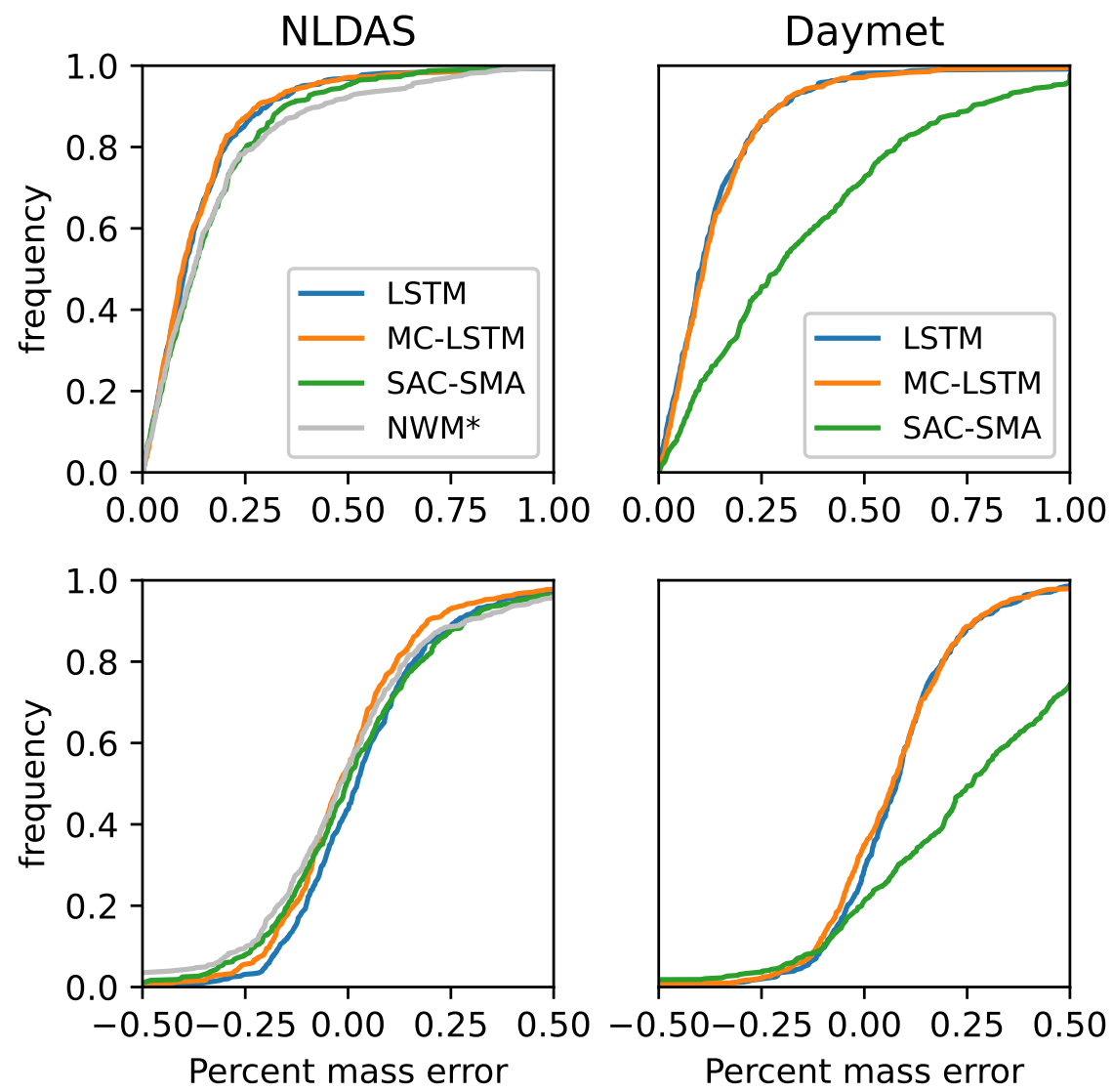

Figure C2. Distribution of mass balance error across the 484 basins. Top: Cumulative distribution curves of the absolute mass error form models forced with NLDAS (left) and Daymet (right). Bottom: Cumulative distributions of mass error from models forced with NLDAS (left) and Daymet (right).

where when the conceptual model (SAC-SMA), the physics-based model (NWM-Rv2), the physics informed ML Model (MC-LSTM) and the pure data driven model (LSTM) all show relatively small to moderate mass bass bias error in the Northeastern CONUS, high mass bias error in the central CONUS and moderate mass bias in west coast regions. The exception to this trend is that the Great Basin has a low mass bias error from LSTM, MC-LSTM and SAC-SMA, but a high mass bias error from NWM. 

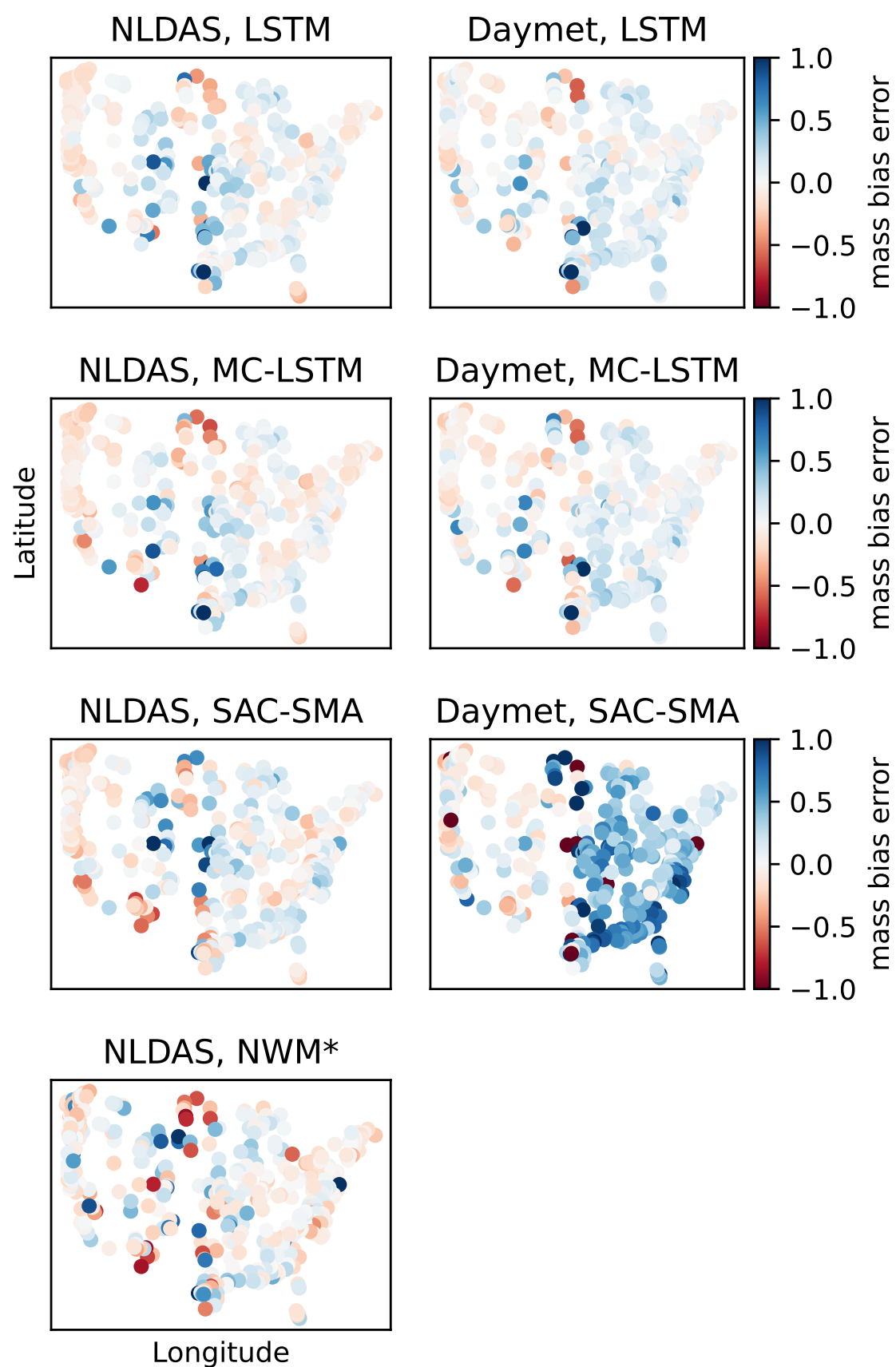

Figure C3. Geospatial distribution of long term positive or negative mass bias error. The left and right columns show the results with NLDAS and Daymet meteorological forcing data, respectively. The four rows are associated (from top to bottom) with LSTM, MC-LSTM, SAC-SMA and NWM. The astrisct (*) on the bottom left sub-plot label indicates that the NWM was not calibrated on the same time period as the LSTM, MC-LSTM and SAC-SMA models. 

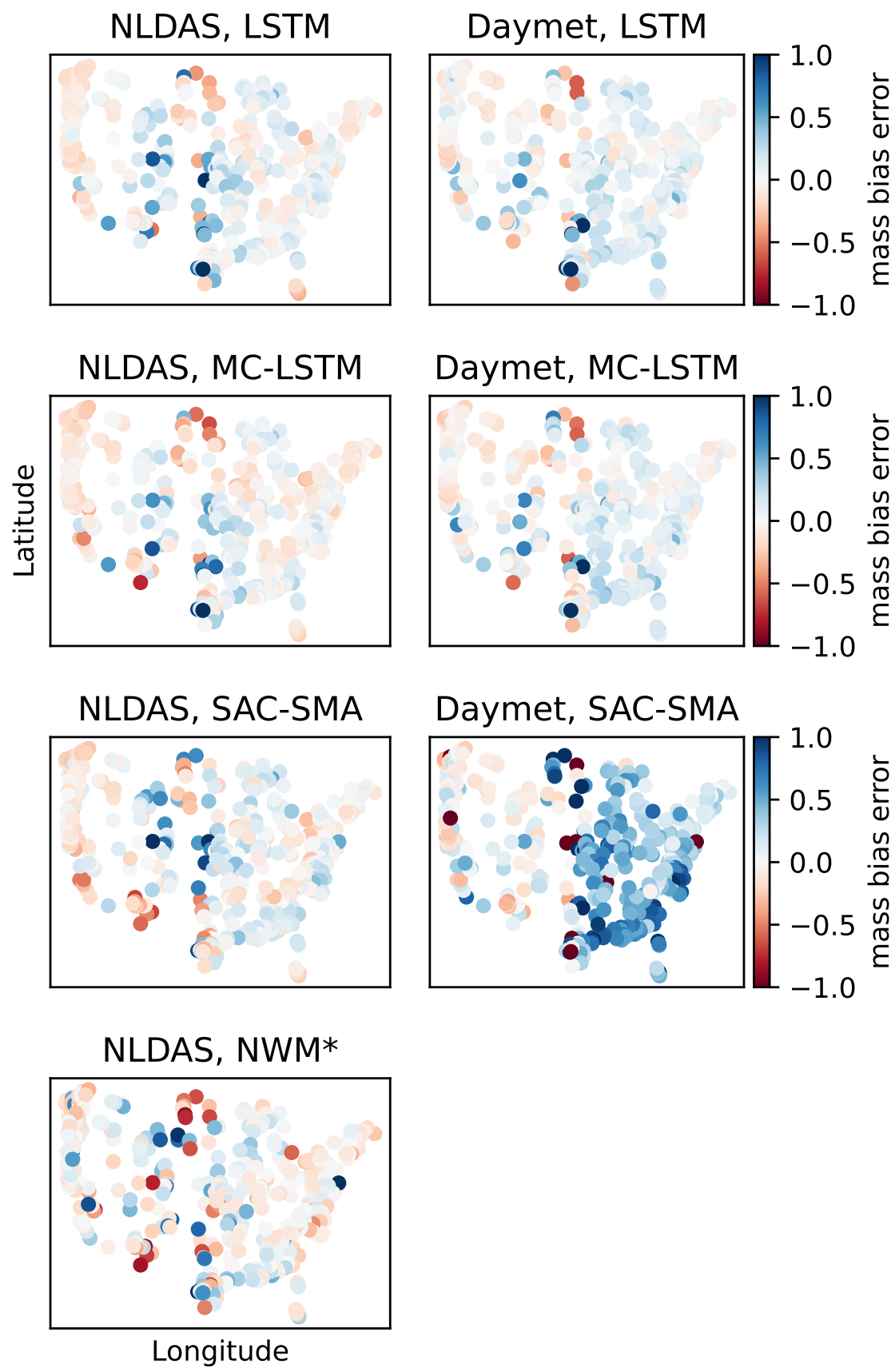

Figure C4. Regional mass balance errors from LSTM, MC-LSTM and SAC-SMA with Daymet forcings. Souris-Red-Rainy region (Hydrologic Unit Code 09) is absent due to a lack of sufficient basins. 


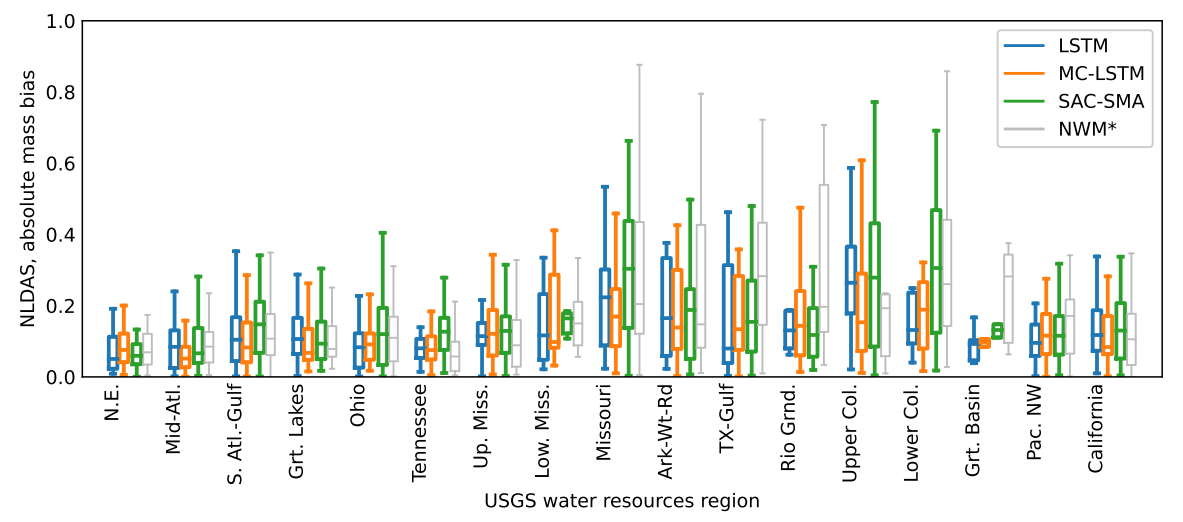

Figure C5. Regional mass balance errors from LSTM, MC-LSTM and SAC-SMA with NLDAS forcings. Souris-Red-Rainy region (Hydrologic Unit Code 09) is absent due to a lack of sufficient basins. The astrict on the NWM label indicates that the model was calibrated on a separate time period than the other three models, and is thus not directly comparable. 